\title{
ENTROPIES FOR RADIALLY SYMMETRIC HIGHER-ORDER NONLINEAR DIFFUSION EQUATIONS*
}

\author{
MARIO BUKAL ${ }^{\dagger}$, ANSGAR JÜNGEL ${ }^{\ddagger}$, AND DANIEL MATTHES ${ }^{\S}$
}

\begin{abstract}
A previously developed algebraic approach to proving entropy production inequalities is extended to deal with radially symmetric solutions for a class of higher-order diffusion equations in multiple space dimensions. In application of the method, novel a priori estimates are derived for the thin-film equation, the fourth-order Derrida-Lebowitz-Speer-Spohn equation, and a sixth-order quantum diffusion equation.
\end{abstract}

Key words. Higher-order diffusion equations, thin-film equation, quantum diffusion model, polynomial decision problem, quantifier elimination.

AMS subject classifications. 35B45, 35G25, 35K55, 76A20, 82C70.

\section{Introduction}

In the last two decades, there has been a growing interest in the mathematical analysis of fourth and higher-order nonlinear diffusion equations. Such equations arise, for instance, in lubrication theory and as models for the electron transport in semiconductors; below, we will briefly review several specific examples and their origins in physics. Rigorous results about the existence of solutions and their qualitative behavior are typically much harder to obtain than in the context of the well-studied secondorder diffusion equations. One of the principal difficulties is the non-applicability of comparison principles. To substitute for this loss, one has to rely on suitable a priori estimates.

In [11], the last two authors have proposed a systematic approach to the derivation of a priori estimates for certain classes of nonlinear evolution equations of even order. This procedure allows one to determine Lyapunov functionals, which we call entropies in the following, and to derive integral bounds from their dissipation, called entropy production inequalities. The developed method has been successfully applied to several equations in one space dimension. The main idea is to translate the procedure of integration by parts - which is the core element in most derivations of a priori estimates - into an algebraic problem about the positivity of polynomials. Roughly speaking, to each evolution equation, a polynomial in the spatial derivatives of the solution is associated, and integration by parts allows one to modify the coefficients of this polynomial. If a suitable change of coefficients can be found that makes the resulting polynomial nonnegative, then this corresponds (formally) to a proof of an a priori estimate on the solutions. The key point is that such polynomial decision

*Received: May 3, 2010; accepted (in revised version): August 17, 2010. Communicated by Martin Burger.

The authors acknowledge partial support from the Austrian Science Fund (FWF), grants P20214 and P22108; the Doctoral Program W8 from the FWF; the German Science Foundation (DFG), grant JU 359/7; the Austria-Croatia Project HR 10/2010; the Austria-France Project FR 07/2010; and the Austria-Spain Project ES 08/2010 of the Austrian Exchange Service (ÖAD).

$\dagger$ Institute for Analysis and Scientific Computing, Vienna University of Technology, Wiedner Hauptstraße 8-10, 1040 Wien, Austria (mbukal@asc.tuwien.ac.at).

${ }^{\ddagger}$ Institute for Analysis and Scientific Computing, Vienna University of Technology, Wiedner Hauptstraße 8-10, 1040 Wien, Austria (juengel@tuwien.ac.at).

$\S$ Institute for Analysis and Scientific Computing, Vienna University of Technology, Wiedner Hauptstraße 8-10, 1040 Wien, Austria (matthes@tuwien.ac.at). 
problems are well-known in real algebraic geometry, and there exist powerful methods to solve them.

The approach of [11] can, in principle, be generalized in a straightforward way to multidimensional higher-order equations by taking all partial derivatives as polynomial variables. However, this leads, even in simple situations, to huge polynomial expressions, and the corresponding algebraic problem is too complex to be solved directly, even with the aid of computer algebra systems. The method has been successfully adapted to deal with certain multidimensional equations of second order $[15,19]$ and fourth order $[12,20]$, but the systematic extension of the scheme to the general multidimensional case is still under development. In this paper, we propose a further adaption that works generally for radially symmetric solutions to higherorder nonlinear equations of a certain homogeneity, and we prove its practicability by applying our scheme to the Equations (1.1)-(1.3) listed below.

Before describing our main results, we briefly review the example equations. The first is the fourth-order thin-film equation

$$
\partial_{t} U+\operatorname{div}\left(U^{\beta} \nabla \Delta U\right)=0
$$

which models the flow of a thin liquid along a solid surface with film height $U(t ; x) \geq 0$ (for $\beta=2$ or $\beta=3$ ) or the thin neck of a Hele-Shaw flow in the lubrication approximation (for $\beta=1$ ). For details, we refer to the reviews [2, 21]. The one-dimensional family of equations has been first analyzed by Bernis and Friedman [1]; for the multidimensional case, we refer to the work of Dal Passo et al. [7] and references therein.

The other examples we are dealing with arise as approximations of the quantum diffusion model by Degond et al. In [8], an equation for the dynamics of the electron density in a plasma has been derived. Although essentially non-local in its nature, the partial pseudo-differential equation can be developed asymptotically in terms of the reduced Planck constant $\hbar$, and this provides a family of approximative (genuine) partial differential equations.

The equation for the electron density $U(t ; x) \geq 0$ obtained at order $\hbar^{2}$ is (after neglecting electric fields)

$$
\partial_{t} U+\operatorname{div}\left(U \nabla\left(\frac{\Delta \sqrt{U}}{\sqrt{U}}\right)\right)=0 .
$$

Interestingly, this equation - in one space dimension - also describes the fluctuations of the interface between the regions of positive and negative particle spins in the Toom model. It has been derived by Derrida et al. in [9]; we shall therefore refer to (1.2) as Derrida-Lebowitz-Speer-Spohn (DLSS) equation in the following. It has been first analyzed in [4] for local positive smooth solutions and then in [14] for global nonnegative weak solutions. The existence of weak solutions to the multidimensional equation was proved recently in $[10,12]$.

When the non-local quantum diffusion model is expanded to order $\hbar^{4}$, the main part of the differential operator is of sixth order, and the corresponding equation reads as

$$
\partial_{t} U-\operatorname{div}\left(U \nabla\left[\sum_{j, k=1}^{d}\left(\frac{1}{2}\left(\partial_{j k}^{2} \log U\right)^{2}+\frac{1}{U} \partial_{j k}^{2}\left(U \partial_{j k}^{2} \log U\right)\right)\right]\right)=0 .
$$

The one-dimensional version of (1.3) has been derived in [11]; see Appendix A for the derivation in the general case. The one-dimensional equations with periodic boundary conditions have been analyzed in [13]. 
The objective of this paper is to prove, for radially symmetric smooth positive solutions $U(t)$ to (1.1), (1.2), or (1.3) satisfying no-flux and Neumann-type boundary conditions (see below for the precise conditions), estimates of the type

$$
\frac{d E_{\alpha}}{d t}[U(t)]+c Q_{\alpha}[U(t)] \leq 0
$$

on a specific range of parameters $\alpha$, where

$$
\begin{aligned}
& E_{\alpha}[U]=\frac{1}{\alpha(\alpha-1)} \int_{\Omega} U^{\alpha} \mathrm{d} x, \quad \alpha \neq 0,1, \\
& E_{0}[U]=\int_{\Omega}(U-\log U) \mathrm{d} x, \\
& E_{1}[U]=\int_{\Omega}(U(\log U-1)+1) \mathrm{d} x .
\end{aligned}
$$

Above, $\Omega=B^{d}=\{|x|<1\}$ is the unit ball in $\mathbb{R}^{d}, c \geq 0$ is a constant independent of the solution $U$, and $Q_{\alpha}$ is a nonnegative functional containing higher-order derivatives of $U$. We call $E_{\alpha}$ an entropy if (1.4) holds with some suitable choice of $Q_{\alpha}$ and $c \geq 0$ for arbitrary solutions $U(t)$ of the evolution equation under consideration. The estimate (1.4) is referred to as an entropy production inequality, and $Q_{\alpha}$ is the corresponding entropy production. Inequalities like (1.4) provide a priori bounds for the evolution; they are a necessary first step in proofs for existence of solutions, and they allow one to describe the equilibration behavior of the solutions.

Entropy production inequalities for the evolution equations reviewed above have been extensively studied in the literature. Concerning the thin-film equation, with no-flux and homogeneous Neumann boundary conditions, it has been shown in $[3,7]$ that $E_{\alpha}$ is an entropy if $3 / 2 \leq \alpha+\beta \leq 3$. The same result holds for periodic boundary conditions [11]. This bound turns out to be sharp, at least in the one-dimensional case [17]. Moreover, the entropy production $Q_{\alpha}$ in (1.4) can be made explicit: a valid choice is $Q_{\alpha}[U]=\int_{\Omega}\left|\left(U^{(\alpha+\beta) / 2}\right)_{x x}\right|^{2} \mathrm{~d} x$ with a suitable $c>0$ if $3 / 2<\alpha+\beta<3$; see [11].

Let $U$ be a smooth solution to the DLSS Equation (1.2) with periodic boundary conditions. Then (1.4) holds with

$$
c=\frac{2 p(\alpha)}{\alpha^{2}(p(\alpha)-p(0))},
$$

where $p(\alpha)=-\alpha^{2}+2 \alpha(d+1) /(d+2)-(d-1)^{2} /(d+2)^{2}$, and $Q_{\alpha}[U]=\int_{\Omega}\left(\Delta U^{\alpha / 2}\right)^{2} \mathrm{~d} x$ for all $0<\alpha<2(d+1) /(d+2)$ [12]. In the one-dimensional case, this estimate holds true for a larger range of values for $\alpha$, with $c=2 / \alpha^{2}$ for $0<\alpha<4 / 3$ and $c=8(3-$ $2 \alpha) / \alpha^{3}$ for $4 / 3<\alpha<3 / 2$.

Entropy estimates for the sixth-order quantum diffusion model (1.3) with periodic boundary conditions are available only in one space dimension. In fact, it has been shown in [13] that $E_{1}$ is an entropy and (1.4) holds for some $c>0$ and with $Q_{1}[U]=$ $\int_{\Omega}\left((\sqrt{U})_{x x x}^{2}+(\sqrt[6]{U})_{x}^{6}\right) \mathrm{d} x$.

To our knowledge, no entropy production inequalities (1.4) are available for the DLSS equation with no-flux and Neumann boundary conditions ${ }^{1}$ and for the sixthorder equation with $\alpha \neq 1$. In this paper, we will prove such results for radially symmetric solutions.

\footnotetext{
${ }^{1}$ In one spatial dimension, calculations related to entropy production estimates typically carry over from one "reasonable" boundary condition to another (e.g. from periodic to no-flux or Neumann conditions). In dimensions $d \geq 2$, this is no longer true since the boundary terms resulting from integration by parts have a more complicated structure.
} 
The advantage of considering radially symmetric solutions $U(t ; x)=u(t ;|x|)$ - in comparison to solutions of the full multidimensional problem - is that the reduced function $u(t ; r)$ satisfies an evolution equation with only one spatial variable $r>0$. Still, the proof of entropy production inequalities (1.4) is substantially more difficult than in the genuinely one-dimensional situation considered before [11]. The reason is that the variable $r$ appears explicitly in the evolution equation. On the algebraic level, this adds one polynomial variable.

In the following we summarize our main results. Below, $\Omega=B^{d} \subset \mathbb{R}^{d}$ denotes the $d$-dimensional unit ball, and $\nu$ is the exterior unit normal vector to $\partial \Omega$.

THEOREM 1.1 (Thin-film equation). Let $U$ be a radially symmetric smooth and positive solution to the thin-film equation with homogeneous Neumann and no-flux boundary conditions:

$$
\begin{cases}\partial_{t} U+\operatorname{div}\left(U^{\beta} \nabla \Delta U\right)=0 & \text { in } \Omega, \text { for } t>0 \\ \nabla U \cdot \nu=U^{\beta} \nabla \Delta U \cdot \nu=0 & \text { on } \partial \Omega, \text { for } t>0 .\end{cases}
$$

Then the functionals $E_{\alpha}$, defined in (1.5), are entropies provided that $3 / 2 \leq \alpha+\beta \leq 3$. In this case, the entropy production inequality (1.4) holds with

$$
c=\frac{16}{(\alpha+\beta)^{4}}(3-\alpha-\beta)(2(\alpha+\beta)-3) \quad \text { and } \quad Q_{\alpha}[U]=\int_{\Omega}\left(\Delta U^{(\alpha+\beta) / 2}\right)^{2} \mathrm{~d} x
$$

The facts that $E_{\alpha}$ is a Lyapunov functional for $3 / 2 \leq \alpha \leq 3$ and that $Q_{\alpha}[U]$ is an entropy production, for some unspecified constant $c$, are well known [7]. The explicit dependence of the constant $c$ on $\alpha$ and $\beta$ is new. This dependence is illustrated in Figure 1.1.

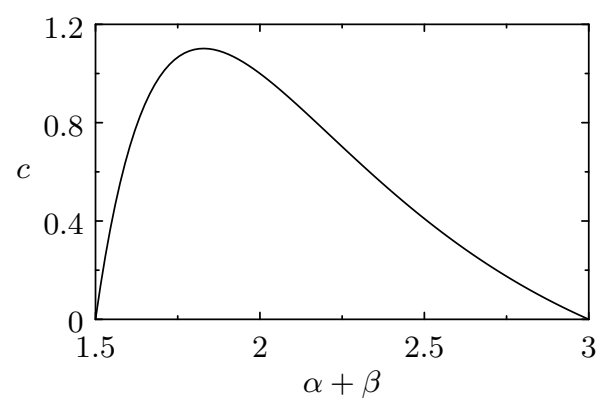

FIG. 1.1. Thin-film equation: Values of $c$ as a function of $\alpha+\beta$.

THEOREM 1.2 (DLSS equation). Let $U$ be a radially symmetric smooth positive solution to the DLSS equation with homogeneous Neumann and no-flux boundary conditions:

$$
\left\{\begin{array}{l}
\partial_{t} U+\operatorname{div}\left(U \nabla\left(\frac{\Delta \sqrt{U}}{\sqrt{U}}\right)\right)=0 \quad \text { in } \Omega, \text { for } t>0 \\
\nabla U \cdot \nu=U \nabla\left(\frac{\Delta \sqrt{U}}{\sqrt{U}}\right) \cdot \nu=0 \quad \text { on } \partial \Omega, \text { for } t>0
\end{array}\right.
$$


Then the functionals $E_{\alpha}$, defined in (1.5), are entropies if

$$
\begin{aligned}
d=1,2,3, \text { or } 4, \text { and } & \frac{(\sqrt{d}-1)^{2}}{d+2} \leq \alpha \leq \frac{3}{2}, \\
d=5,6, \text { or } 7, \text { and } & \frac{(\sqrt{d}-1)^{2}}{d+2} \leq \alpha \leq \frac{(\sqrt{d}+1)^{2}}{d+2}, \\
d \geq 8 \text { and } & \frac{d-4}{2(d-2)} \leq \alpha \leq \frac{(\sqrt{d}+1)^{2}}{d+2},
\end{aligned}
$$

and the entropy production inequality (1.4) holds with $Q_{\alpha}[U]=\int_{\Omega}\left(\Delta U^{\alpha / 2}\right)^{2} \mathrm{~d} x$ and

$$
\begin{aligned}
d=1,2,3: \quad c=\left\{\begin{array}{lr}
\frac{2 p(\alpha)}{\alpha^{2}(p(\alpha)-p(0))} & \text { for } \frac{(\sqrt{d}-1)^{2}}{d+2}<\alpha \leq \frac{5 d+7}{3 d+6}, \\
\frac{8(3-2 \alpha)}{\alpha^{3}} & \text { for } \frac{5 d+7}{3 d+6}<\alpha<\frac{3}{2},
\end{array}\right. \\
d=4,5,6,7: \quad c=\frac{2 p(\alpha)}{\alpha^{2}(p(\alpha)-p(0))} \\
d \geq 8: \quad c= \begin{cases}\frac{16(d-2) \alpha-8(d-4)}{d^{2} \alpha^{3}} & \text { for } \frac{(\sqrt{d}-1)^{2}}{d+2}<\alpha<\frac{(\sqrt{d}+1)^{2}}{d+2}, \\
\frac{2 p(\alpha)}{2(d-2)}<\alpha \leq \frac{d^{2}-5 d-8}{d^{2}-2 d-8},\end{cases}
\end{aligned}
$$

where $p(\alpha)=-\alpha^{2}+2 \alpha(d+1) /(d+2)-(d-1)^{2} /(d+2)^{2}$.

The dependence of $c$ on $\alpha$ is illustrated in Figure 1.2 for various dimensions $d$. The values for $c$ for $d=4,5,6,7$ are the same as those derived in [12]. We are able to improve the results from [12] in the radially symmetric case for space dimensions $d=2,3$ and $d \geq 8$, see Figure 1.3. Our main contribution is that the range of parameters $\alpha$ leading to entropies is larger than in [12].

It is known from [11] that the bounds $0 \leq \alpha \leq 3 / 2$ are optimal if $d=1$. We prove in Section 5 that in dimension $d=2$, no entropies exist for $\alpha \leq 0$, and that the lower bound $\alpha=(d-4) /(2 d-4)$ is optimal for $d \geq 8$.
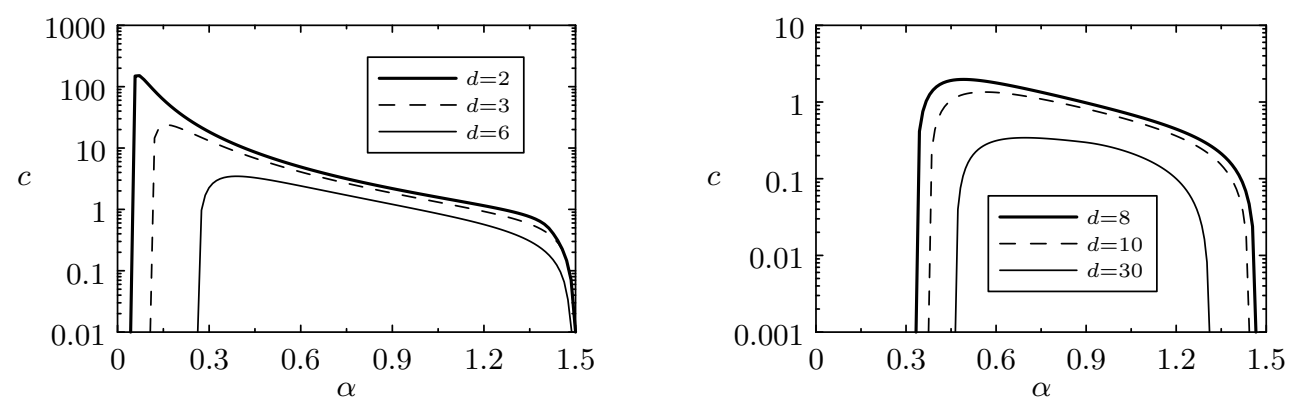

FIG. 1.2. DLSS equation: Values of $c$ as a function of $d$ and $\alpha$. 

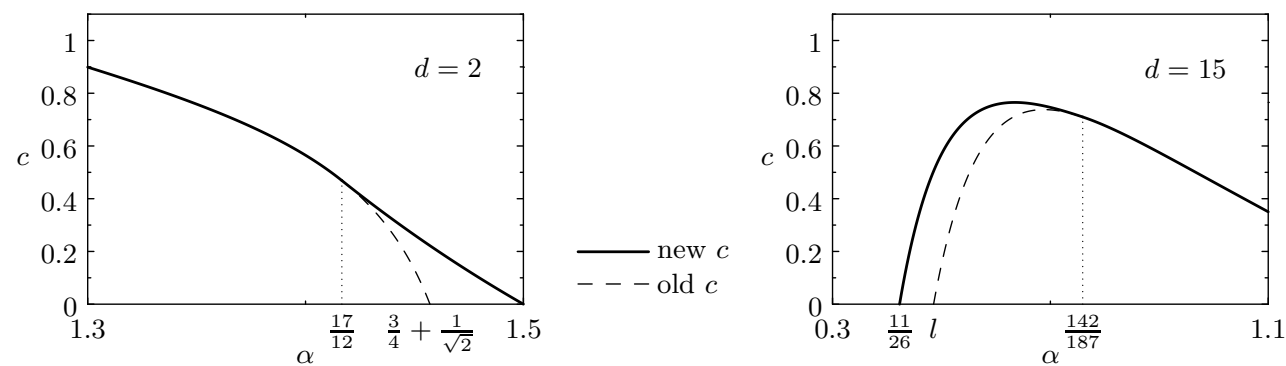

FIG. 1.3. DLSS equation: Values of $c$ as a function of $\alpha$. The solid line represents the values from Theorem 1.2, the dashed line those from [12]. Here, $l=2(8-\sqrt{15}) / 17$.

TheOrem 1.3 (Sixth-order quantum diffusion equation). Let $U$ be a radially symmetric smooth and positive solution to the sixth-order quantum diffusion equation:

$$
\left\{\begin{aligned}
& \partial_{t} U-\operatorname{div}\left(U \nabla\left[\sum_{j, k=1}^{d}\left(\frac{1}{2}\left(\partial_{j k}^{2} \log U\right)^{2}+\frac{1}{U} \partial_{j k}^{2}\left(U \partial_{j k}^{2} \log U\right)\right)\right]\right)=0 \quad \text { in } \Omega, \text { for } t>0, \\
& \nabla U \cdot \nu=U \nabla\left(\frac{\Delta \sqrt{U}}{\sqrt{U}}\right) \cdot \nu \\
&=U \nabla\left[\sum_{j, k=1}^{d}\left(\frac{1}{2}\left(\partial_{j k}^{2} \log U\right)^{2}+\frac{1}{U} \partial_{j k}^{2}\left(U \partial_{j k}^{2} \log U\right)\right)\right] \cdot \nu=0 \text { on } \partial \Omega .
\end{aligned}\right.
$$

Then the functionals $E_{\alpha}$, defined in (1.5), are entropies if

$$
\begin{array}{ll}
d=1 \text { and } & 0.1927 \ldots \leq \alpha \leq 1.1572 \ldots, \\
d=2 \text { and } & 0.2827 \ldots \leq \alpha \leq 1.0982 \ldots, \\
d=3 \text { and } & 0.3470 \ldots \leq \alpha \leq 1.0517 \ldots \\
d=4 \text { and } & 0.3968 \ldots \leq \alpha \leq 1.0123 \ldots \\
d=5 \text { and } & 0.4380 \ldots \leq \alpha \leq 0.9775 \ldots
\end{array}
$$

Moreover, in dimensions $d=1, \ldots, 4$ and for $\alpha=1$, the entropy production inequality (1.4) holds for some $c>0$ if one chooses

$$
Q_{1}[U]=\int_{\Omega}\left(|\nabla \Delta \sqrt{U}|^{2}+|\nabla \sqrt[6]{u}|^{6}\right) \mathrm{d} x
$$

The bounds for $\alpha$ are roots of certain polynomials and can be determined only numerically; see Figure 1.4. The Lyapunov property of $E_{\alpha}$ for $\alpha=1$ and $d=1$ is proved in [13]. The proof of this property for $\alpha \neq 1$ and $d>1$ as well as the entropy production inequality are new. Interestingly, it seems that the logarithmic functional $E_{1}$ is no longer a Lyapunov functional for the sixth-order equation in (the unphysical) space dimensions higher than 4 . We remark that in dimension $d=2$, the results from Section 5 show that there are no entropies if $\alpha>4 / 3$.

The paper is organized as follows. The algebraic formalism is developed in Section 2. Section 3.2 is devoted to the proof of two auxiliary results about quadratic polynomials. The proofs for Theorems 1.1 to 1.3 are given in Section 4. In Section 


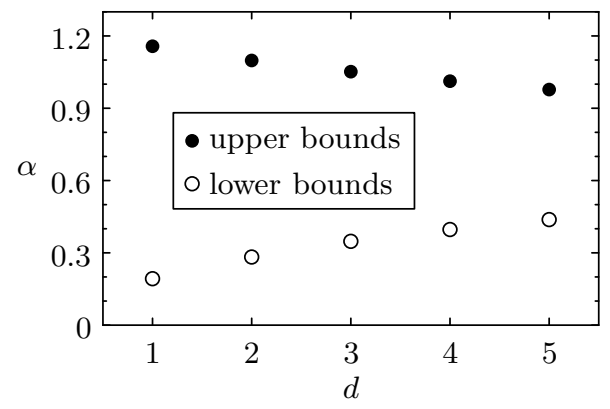

FIG. 1.4. Sixth-order quantum diffusion equation: Upper and lower bounds for $\alpha$ depending on the dimension $d$.

5 , a sufficient condition is provided under which $E_{\alpha}$ is not an entropy. Finally, the derivation of the quantum diffusion Equation (1.3) from the Wigner-BGK model is outlined in Appendix A and a Mathematica notebook used in the proof of Theorem 1.3 is given in Appendix B.

\section{Decision problem and shift polynomials}

In this section, we establish the connection between the analytical problem of proving entropy production inequalities (1.4) and an algebraic problem about the non-negativity of certain polynomials. This correspondence - which is summarized in Lemma 2.1 below - constitutes an extension of the ideas previously developed for entropy estimates in one spatial dimension by the last two authors [11]; see also [17] for an alternative approach. The proof of the main theorems are then obtained by solution of the associated algebraic problems.

2.1. Formulation as a decision problem. To start with, we need to introduce some notation. First, observe that $U: \bar{\Omega} \rightarrow \mathbb{R}_{+}$is a smooth and positive radially symmetric function if and only if there exists some

$$
u \in \mathcal{U}:=\left\{u \in C^{\infty}\left([0,1] ; \mathbb{R}_{+}\right)\left|\partial_{r}^{m}\right|_{r=0} u(r)=0 \text { for all odd } m \in \mathbb{N}\right\}
$$

such that $U(x)=u(r)$ with $r=|x|$ for all $x \in \Omega=B^{d}$. We shall refer to $u$ as the (radial) reduction of $U$, and to $U$ as the (radially symmetric) extension of $u$.

Throughout this article, $\eta$ and $\xi_{1}, \xi_{2}, \ldots$ denote real variables. For $k \in \mathbb{N}$, let $\Sigma_{k}$ be the linear span of all monomials $\eta^{s} \xi_{1}^{p_{1}} \cdots \xi_{k}^{p_{k}}$ satisfying $s+1 \cdot p_{1}+\cdots+k \cdot p_{k}=k$. Alternatively, one can define $\Sigma_{k}$ as the set of polynomials $P$ in $\left(\eta, \xi_{1}, \ldots, \xi_{k}\right)$ with the homogeneity property

$$
P\left(\lambda \eta, \lambda \xi_{1}, \lambda^{2} \xi_{2}, \ldots, \lambda^{k} \xi_{k}\right)=\lambda^{k} P\left(\eta, \xi_{1}, \xi_{2}, \ldots, \xi_{k}\right)
$$

for all $\lambda \in \mathbb{R}$. To any $P \in \Sigma_{k}$, we associate a non-linear differential operator $\mathrm{D}_{P}$ of order less or equal to $k$ by

$$
\mathrm{D}_{P}[u, r]=P\left(\frac{1}{r}, \frac{\partial_{r} u}{u}(r), \ldots, \frac{\partial_{r}^{k} u}{u}(r)\right)
$$

acting on functions $u \in \mathcal{U}$. 
The key point behind this formalism is that the reductions $u(t ; r)$ of radially symmetric solutions $U(t ; x)$ to the evolution equations under consideration satisfy equations of the form

$$
\partial_{t} u+r^{-(d-1)} \partial_{r}\left(r^{d-1} u^{\beta+1} \mathrm{D}_{P}[u, r]\right)=0, \quad t>0,
$$

where $\beta \in \mathbb{R}$ is a parameter, $P \in \Sigma_{K-1}$, and $K$ is the order of the equation.

EXAMPLE 2.1. Recall the representation of the gradient, divergence and Laplacian in radial coordinates: If $W(x)=w(r)$ is a radially symmetric function on $\Omega=B^{d}$, and $\mathbf{e}_{r}=x / r$ is the unit vector in radial direction, then

$$
\nabla_{x} W(x)=w_{r}(r) \mathbf{e}_{r}, \quad \operatorname{div}_{x}\left(W(x) \mathbf{e}_{r}\right)=w_{r}(r)+\frac{d-1}{r} w(r)=r^{-(d-1)} \partial_{r}\left(r^{d-1} w(r)\right),
$$

and, in combination,

$$
\Delta_{x} W(x)=w_{r r}(r)+\frac{d-1}{r} w_{r}(r)=: \Delta_{r} w(r)
$$

For our examples, this leads to the following:

(A) A radially symmetric solution $U(t ; x)=u(t ; r)$ to the thin-film Equation (1.1) satisfies:

$$
\partial_{t} U=-\operatorname{div}_{x}\left(U^{\beta} \nabla_{x} \Delta_{x} U\right)=-\operatorname{div}_{x}\left[u^{\beta+1}\left(\frac{u_{r r r}}{u}+\frac{d-1}{r}\left(\frac{u_{r r}}{u}-\frac{u_{r}}{r u}\right)\right) \mathbf{e}_{r}\right] .
$$

This equation is of the form (2.2), with

$$
P(\eta, \xi)=\xi_{3}+(d-1)\left(\eta \xi_{2}-\eta^{2} \xi_{1}\right) .
$$

(B) A radially symmetric solution to the DLSS Equation (1.2) satisfies:

$$
\begin{aligned}
\partial_{t} U & =-\operatorname{div}_{x}\left[U \nabla_{x}\left(\frac{\Delta_{x} \sqrt{U}}{\sqrt{U}}\right)\right] \\
& =-\operatorname{div}_{x}\left[u \partial_{r}\left(\frac{(\sqrt{u})_{r r}}{\sqrt{u}}+\frac{d-1}{r} \frac{(\sqrt{u})_{r}}{\sqrt{u}}\right) \mathbf{e}_{r}\right] \\
& =-\operatorname{div}_{x}\left[u\left(\frac{1}{2} \frac{u_{r r r}}{u}-\frac{u_{r r}}{u} \frac{u_{r}}{u}+\frac{1}{2} \frac{u_{r}^{3}}{u^{3}}+\frac{d-1}{2 r}\left(\frac{u_{r r}}{u}-\frac{u_{r}^{2}}{u^{2}}-\frac{u_{r}}{r u}\right)\right) \mathbf{e}_{r}\right] .
\end{aligned}
$$

Also this equation is of the form (2.2), with $\beta=0$ and

$$
P(\eta, \xi)=\frac{1}{2}\left(\xi_{3}-2 \xi_{2} \xi_{1}+\xi_{1}^{3}+(d-1)\left(\eta \xi_{2}-\eta \xi_{1}^{2}-\eta^{2} \xi_{1}\right)\right) .
$$

(C) If $U(t ; x)=u(t ; r)$ is a radially symmetric solution to the sixth order Equation (1.3), then tedious but straightforward computations show that $\partial_{t} U=$ 
$\operatorname{div}_{x}\left(u G(u) \mathbf{e}_{r}\right)$, where

$$
\begin{aligned}
G(u)= & -6 \frac{u_{r}^{5}}{u^{5}}+18 \frac{u_{r}^{3}}{u^{3}} \frac{u_{r r}}{u}-11 \frac{u_{r}}{u} \frac{u_{r r}^{2}}{u^{2}}-8 \frac{u_{r}^{2}}{u^{2}} \frac{u_{r r r}}{u}+3 \frac{u_{r}}{u} \frac{u_{r r r r}}{u} \\
& +5 \frac{u_{r r}}{u} \frac{u_{r r r}}{u}-\frac{u_{r r r r r}}{u} \\
& -(d-1) \frac{1}{r}\left(-6 \frac{u_{r}^{4}}{u^{4}}+(2 d-7) \frac{1}{r} \frac{u_{r}^{3}}{u^{3}}+14 \frac{u_{r}^{2}}{u^{2}} \frac{u_{r r}}{u}+(3 d-8) \frac{1}{r^{2}} \frac{u_{r}^{2}}{u^{2}}-4 \frac{u_{r r}^{2}}{u^{2}}\right. \\
& -3(d-4) \frac{1}{r} \frac{u_{r}}{u} \frac{u_{r r}}{u}-6 \frac{u_{r}}{u} \frac{u_{r r r}}{u}+3(d-3) \frac{1}{r^{3}} \frac{u_{r}}{u}-3(d-3) \frac{1}{r^{2}} \frac{u_{r r}}{u} \\
& \left.+(d-5) \frac{1}{r} \frac{u_{r r r}}{u}+2 \frac{u_{r r r r}}{u}\right) .
\end{aligned}
$$

In principle, one can easily deduce the correct choice of $P$ from here.

Equation (2.2) is supplemented by initial conditions at $t=0$,

$$
u(0, r)=u_{0}(r) .
$$

For the fourth order equations $(K=4)$, homogeneous Neumann and no-flux boundary conditions are assumed,

$$
u_{r}(r)=0, \quad r^{d-1} \mathrm{D}_{P}[u, r]=0 \quad \text { at } r=0 \text { and } r=1 .
$$

Additional boundary conditions will be specified for the sixth order Equation (1.3), when $K=6$.

Notice that the Neumann condition at $r=0$ is already implied by $u \in \mathcal{U}$. On the other hand, the no-flux condition at $r=0$ is in general not trivially satisfied since $\mathrm{D}_{P}[u, r]$ might contain terms with negative powers of $r$. More precisely, the condition is that

$$
\lim _{r \downarrow 0}\left(r^{d-1} \mathrm{D}_{P}[u, r]\right)=0 .
$$

In terms of the radially symmetric extension $U(t ; x)=u(t ; r)$, the homogeneous Neumann and no-flux boundary conditions (2.4) for an equation of the form $\partial_{t} U=$ $\operatorname{div}_{x}\left(U^{\beta+1} G(U)\right)$ correspond to

$$
\nu \cdot \nabla U(t ; x)=0, \quad \nu \cdot U^{\beta+1} G(U)=0 \quad \text { for all } x \in \partial \Omega \text { and } t>0,
$$

with $\nu=\mathbf{e}_{r}$ denoting the outer normal vector at the boundary of the unit sphere.

For radially symmetric solutions $U(t ; x)=u(t ; r)$, the entropy functionals in (1.5) become

$$
E_{\alpha}[U(t)]=\frac{\omega_{d}}{\alpha(\alpha-1)} \int_{0}^{1} u(t ; r)^{\alpha} r^{d-1} \mathrm{~d} r
$$

where $\omega_{d}$ is the surface of the unit sphere in $\mathbb{R}^{d}$. For the time derivative along (2.2), one calculates

$$
\begin{aligned}
\frac{d}{d t} E_{\alpha}[U(t)] & =\frac{\omega_{d}}{\alpha-1} \int_{0}^{1} u(t ; r)^{\alpha-1} \partial_{t} u(t ; r) r^{d-1} d r \\
& =-\omega_{d} \int_{0}^{1} u^{\alpha+\beta}\left(-\frac{\partial_{r} u}{u}\right) P\left(\frac{1}{r}, \frac{\partial_{r} u}{u}, \ldots, \frac{\partial_{r}^{K-1} u}{u}\right) r^{d-1} \mathrm{~d} r
\end{aligned}
$$


where the no-flux boundary conditions in (2.4) have been taken into account. The integrand in (2.5) is again of polynomial structure: defining $S_{0} \in \Sigma_{K}$ by

$$
S_{0}(\eta, \xi)=-\xi_{1} P\left(\eta, \xi_{1}, \ldots, \xi_{K-1}\right),
$$

one can write

$$
\frac{d}{d t} E_{\alpha}[U(t)]=-\omega_{d} I_{0}[u(t)] \quad \text { with } \quad I_{0}[u(t)]:=\int_{0}^{1} u(t ; r)^{\alpha+\beta} \mathrm{D}_{S_{0}}[u(t), r] r^{d-1} \mathrm{~d} r .
$$

Following [11], we call $S_{0}$ the canonical symbol that characterizes the dissipation of $E_{\alpha}$ by $(2.2)$.

Recall that the primary goal is to identify - for a given equation of the form (2.2) - those entropies $E_{\alpha}$ which are monotone in time along all smooth radially symmetric solutions. Thus, we wish to determine values $\alpha \in \mathbb{R}$ such that the corresponding functional $I_{0}$ in (2.6) is nonnegative on $\mathcal{U}$. To prove nonnegativity, we apply integration by parts to the integral expression for $I_{0}$ in a systematic way that we explain now.

Let $\gamma \in \mathbb{R}$ and a polynomial $R \in \Sigma_{K-1}$ be given. Introduce the divergence $T=\delta_{\gamma} R$ as the unique element $T \in \Sigma_{K}$ which satisfies

$$
\partial_{r}\left(r^{d-1} u(r)^{\gamma} \mathrm{D}_{R}[u, r]\right)=r^{d-1} u(r)^{\gamma} \mathrm{D}_{T}[u, r]
$$

for all $u \in \mathcal{U}$. Formally, $\delta_{\gamma}: \Sigma_{K-1} \rightarrow \Sigma_{K}$ is a linear map that acts on monomials $R(\eta, \xi)=\eta^{s} \xi_{1}^{p_{1}} \cdots \xi_{K-1}^{p_{K-1}}$ as follows,

$$
\delta_{\gamma} R(\eta, \xi)=\left[(d-1-s) \eta+\left(\gamma-p_{1}-\cdots-p_{K-1}\right) \xi_{1}+p_{1} \frac{\xi_{2}}{\xi_{1}}+\cdots+p_{K-1} \frac{\xi_{K}}{\xi_{K-1}}\right] R(\eta, \xi) .
$$

For $S=S_{0}+T$ with $T=\delta_{\gamma} R$, where $\gamma=\alpha+\beta$ and $R \in \Sigma_{K-1}$, it follows by the fundamental theorem of calculus that

$$
\begin{aligned}
I[u]:=\int_{0}^{1} u(r)^{\alpha+\beta} \mathrm{D}_{S}[u, r] r^{d-1} \mathrm{~d} r & =\int_{0}^{1} u(r)^{\alpha+\beta}\left(\mathrm{D}_{S_{0}}[u, r]+\mathrm{D}_{T}[u, r]\right) r^{d-1} \mathrm{~d} r \\
& =I_{0}[u]+\left[u(r)^{\alpha+\beta} \mathrm{D}_{R}[u, r] r^{d-1}\right]_{r=0}^{r=1} .
\end{aligned}
$$

Assuming that $u$ satisfies boundary conditions which imply in particular that

$$
r^{d-1} \mathrm{D}_{R}[u, r]=0 \quad \text { at } r=1 \text { and for } r \downarrow 0,
$$

then $I[u]=I_{0}[u]$, i.e., the replacement $S_{0} \mapsto S=S_{0}+T$ modifies the integrand but does not change the value of the integral. Hence, if there exists an $R \in \Sigma_{K-1}$ for which $S=S_{0}+\delta_{\alpha+\beta} R$ is a nonnegative polynomial, then it follows that $I_{0}[u]=I[u]$ is nonnegative for all $u \in \mathcal{U}$ that satisfy (2.8). Consequently, if the boundary conditions (2.4) for (2.2) imply (2.8), then $E_{\alpha}[U(t)]$ is monotone in time for all smooth radially symmetric solutions.

In practice, it is more convenient to work directly with the polynomials $T=$ $\delta_{\gamma} R \in \Sigma_{K}$ rather than with their pre-images $R \in \Sigma_{K-1}$. Let $R_{1}$ to $R_{m}$ be a collection of linearly independent polynomials in $\Sigma_{K-1}$ for which (2.8) holds; we refer to Section 2.2 below for details on how to select appropriate $R$ 's. Denote by $T_{1}=\delta_{\gamma} R_{1}$ to $T_{m}=$ 
$\delta_{\gamma} R_{m}$ their respective divergences, which can be explicitly calculated using the rule (2.7) above. In analogy to [11], we call them shift polynomials. In conclusion of our discussion, the following is now obvious.

LEMma 2.1. If the algebraic decision problem

$$
\exists c_{1}, \ldots, c_{m} \in \mathbb{R}: \forall(\eta, \xi) \in \mathbb{R}^{K+1}:\left(S_{0}+c_{1} T_{1}+\cdots+c_{m} T_{m}\right)(\eta, \xi) \geq 0
$$

can be solved affirmatively, then $E_{\alpha}$ is a Lyapunov functional for (2.2).

Algebraic decision problems of the type (2.9) are solvable in an algorithmic way; this is discussed in Section 3 below. We remark that it would suffice to prove (2.9) for all $\xi \in \mathbb{R}^{K}$ and positive $\eta \in \mathbb{R}$ only, since $\eta=1 / r>0$. However, since both $S_{0}$ and the $T_{j}$ satisfy the homogeneity property $(2.1)$ with an even $K$, their values at $(\eta, \xi)$ and $(-\eta,-\xi)$ agree; thus, (2.9) is true under the restriction $\eta>0$ if and only if it is true without this restriction. We prefer to work directly with (2.9).

2.2. Determination of the shift polynomials. The next goal is the following. For the boundary conditions at $\partial \Omega$ as prescribed in Theorems 1.1 to 1.3 , we shall compose a list of linearly independent shift polynomials $T \in \Sigma_{K}$. Recall that shift polynomials are divergencies $T=\delta_{\gamma} R$ of polynomials $R \in \Sigma_{K-1}$ satisfying the relations (2.8). Consequently, the key is to characterize these $R$ in a systematic way and select, among all of them, those which satisfy (2.8) in all dimensions $d \geq 1$ and give rise to "useful" (in a specific sense explained below) shift polynomials.

To begin with, we discuss the case $K=4$ of the DLSS and the thin film equation. First, we use that fact that $u \in \mathcal{U}$ satisfies homogeneous Neumann boundary conditions,

$$
u_{r}(0)=u_{r}(1)=0 .
$$

We wish to find all polynomials $R \in \Sigma_{K-1}=\Sigma_{3}$ for which (2.8) holds. To this end, observe that

$$
\left.\left(r^{d-1} \mathrm{D}_{R}[u, r]\right)\right|_{r=1}=R\left(1,0, \frac{u_{r r}(1)}{u(1)}, \ldots\right) .
$$

Observe further that $R\left(1,0, \xi_{2}, \xi_{3}\right)=0$ for arbitrary $\xi_{2}$ and $\xi_{3}$ if and only if $R$ can be factored in the form $R\left(\eta, \xi_{1}, \xi_{2}, \xi_{3}\right)=\xi_{1} Q\left(\eta, \xi_{1}, \xi_{2}\right)$ with some $Q \in \Sigma_{2}$. Among polynomials $R$ of this type, it remains to single out those for which also

$$
\lim _{r \downarrow 0}\left(r^{d-1} \mathrm{D}_{R}[u, r]\right)=0 .
$$

Since $\Sigma_{2}$ is spanned by $\xi_{2}, \xi_{1}^{2}, \eta \xi_{1}$, and $\eta^{2}$, we need to investigate (2.11) for $R_{1}=\xi_{1} \xi_{2}$, $R_{2}=\xi_{1}^{3}, R_{3}=\eta \xi_{1}^{2}$, and $R_{4}=\eta^{2} \xi$, respectively. Since $R_{1}$ and $R_{2}$ are independent of $\eta$, both satisfy (2.11). Further, by l'Hospital's rule, and since $u_{r}(0)=0$ and $d \geq 1$,

$$
\begin{aligned}
& \lim _{r \downarrow 0}\left(r^{d-1} \mathrm{D}_{R_{3}}[u, r]\right)=\lim _{r \downarrow 0}\left(\frac{u_{r}(r)}{r} \frac{r^{d-1} u_{r}(r)}{u(r)^{2}}\right)=\frac{u_{r}(0) u_{r r}(0)}{u(0)^{2}} \lim _{r \downarrow 0} r^{d-1}=0, \\
& \lim _{r \downarrow 0}\left(r^{d-1} \mathrm{D}_{R_{4}}[u, r]\right)=\lim _{r \downarrow 0}\left(\frac{u_{r}(r)}{r} \frac{r^{d-2}}{u(r)^{2}}\right)=\frac{u_{r r}(0)}{u(0)} \lim _{r \downarrow 0} r^{d-2} .
\end{aligned}
$$

The second limit does not vanish in dimensions $d=1$ and $d=2$. Therefore, we shall not use $R_{4}$ for further computations. 
According to (2.7), the corresponding shift polynomials are

$$
\begin{aligned}
& T_{1}(\eta, \xi)=\delta_{\alpha+\beta} R_{1}(\eta, \xi)=(\alpha+\beta-2) \xi_{1}^{2} \xi_{2}+\xi_{1} \xi_{3}+\xi_{2}^{2}+(d-1) \eta \xi_{1} \xi_{2} \\
& T_{2}(\eta, \xi)=\delta_{\alpha+\beta} R_{2}(\eta, \xi)=(\alpha+\beta-3) \xi_{1}^{4}+3 \xi_{1}^{2} \xi_{2}+(d-1) \eta \xi_{1}^{3} \\
& T_{3}(\eta, \xi)=\delta_{\alpha+\beta} R_{3}(\eta, \xi)=(\alpha+\beta-2) \eta \xi_{1}^{3}+(d-2) \eta^{2} \xi_{1}^{2}+2 \eta \xi_{1} \xi_{2} .
\end{aligned}
$$

This finishes the discussion of the homogeneous Neumann boundary conditions (2.10) for equations of order $K=4$.

Next, we continue to assume $K=4$, and we recall that $u \in \mathcal{U}$ also satisfies no-flux boundary conditions, i.e.,

$$
r^{d-1} \mathrm{D}_{P}[u, r]=0 \quad \text { at } r=0 \text { and } r=1
$$

with the corresponding polynomials $P \in \Sigma_{3}$ given in Example 2.1 (A) and (B). Thus, trivially, $P$ itself satisfies $(2.8)$, giving rise to the shift polynomial $T_{4}=\delta_{\alpha+\beta} P$. However, it is easily seen that $T_{4}$ is of no use for our calculations: The coefficient of $\xi_{3}$ in the polynomial $P$ is positive, so the coefficient of $\xi_{4}$ in $T_{4}$ is positive as well. Recalling that $S_{0}=-\xi_{1} P$ does not contain $\xi_{4}$ at all, it follows that $S=S_{0}+c_{4} T_{4}$ diverges to $-\infty$ as $\xi_{4} \rightarrow \pm \infty$ if $c_{4} \lessgtr 0$ (keeping $\eta, \xi_{1}, \xi_{2}$ and $\xi_{3}$ fixed). Hence, for $S=S_{0}+c_{4} T_{4}$ to have a definite sign, it is required that $c_{4}=0$. Consequently, we omit $T_{4}$ in the following.

REMARK 2.2. The argument is not completely conclusive, since there could be another shift polynomial $T_{5} \in \Sigma_{4}$ for which $T_{4}+T_{5}$ is non-trivial and does not contain $\xi_{4}$. In fact, this cannot happen in the context of radially symmetric solutions, but cancellations of this type do occur when dealing with general multi-dimensional solutions.

We turn to the sixth-order Equation (1.3) and start again with the discussion of homogeneous Neumann boundary conditions (2.10). Arguing as for $K=4$ above, it suffices to consider polynomials $R$ of the form $R_{i}(\xi, \eta)=\xi_{1} Q(\xi, \eta)$ with $Q \in \Sigma_{K-2}=\Sigma_{4}$, There are 12 such polynomials, listed in Table 2.1 below.

REMARK 2.3. Observe that the 5 -tuples $\left(p_{1}, \ldots, p_{5}\right)$ in the table represent precisely the integer partitions of $5-s$ with $p_{1} \geq 1$. Generally, for a differential operator of order $K$, one would find $(K-1)$-tuples of integer partitions. This indicates the rapid growth of the number of shift polynomials with $K$.

We investigate the limits $(2.11)$ corresponding to these $R_{i}$. For $R_{8}=\eta \xi_{1} \xi_{3}$, $R_{9}=\eta^{2} \xi_{1}^{3}, R_{10}=\eta^{2} \xi_{1} \xi_{2}, R_{11}=\eta^{3} \xi_{1}^{2}$, and $R_{12}=\eta^{4} \xi_{1}$, respectively, one obtains by l'Hospital's rule (using that $u_{r}(0)=u_{r r r}(0)=0$ for all $u \in \mathcal{U}$ ) that

$$
\begin{gathered}
\lim _{r \downarrow 0}\left(r^{d-1} \mathrm{D}_{R_{8}}[u, r]\right)=\lim _{r \downarrow 0}\left(\frac{u_{r}(r)}{r} \frac{r^{d-1} u_{r r r}(r)}{u(r)^{2}}\right)=\frac{u_{r r}(0) u_{r r r r}(0)}{u(0)^{2}} \lim _{r \downarrow 0} r^{d}=0, \\
\lim _{r \downarrow 0}\left(r^{d-1} \mathrm{D}_{R_{9}}[u, r]\right)=\lim _{r \downarrow 0}\left(\frac{u_{r}(r)^{3}}{r^{3}} \frac{r^{d}}{u(r)^{3}}\right)=\left(\frac{u_{r r}(0)}{u(0)}\right)^{3} \lim _{r \downarrow 0} r^{d}=0, \\
\lim _{r \downarrow 0}\left(r^{d-1} \mathrm{D}_{R_{10}}[u, r]\right)=\lim _{r \downarrow 0}\left(\frac{u_{r}(r)}{r} \frac{r^{d-2} u_{r r}(r)}{u(r)^{2}}\right)=\left(\frac{u_{r r}(0)}{u(0)}\right)^{2} \lim _{r \downarrow 0} r^{d-2}, \\
\lim _{r \downarrow 0}\left(r^{d-1} \mathrm{D}_{R_{11}}[u, r]\right)=\lim _{r \downarrow 0}\left(\frac{u_{r}(r)^{2}}{r^{2}} \frac{r^{d-2}}{u(r)^{2}}\right)=\left(\frac{u_{r r}(0)}{u(0)}\right)^{2} \lim _{r \downarrow 0} r^{d-2}, \\
\lim _{r \downarrow 0}\left(r^{d-1} \mathrm{D}_{R_{12}}[u, r]\right)=\lim _{r \downarrow 0}\left(\frac{u_{r}(r)}{r} \frac{r^{d-4}}{u(r)}\right)=\frac{u_{r r}(0)}{u(0)} \lim _{r \downarrow 0} r^{d-4} .
\end{gathered}
$$




\begin{tabular}{c|cccccc}
$\#$ & $s$ & $p_{1}$ & $p_{2}$ & $p_{3}$ & $p_{4}$ & $p_{5}$ \\
\hline 1 & 0 & 5 & 0 & 0 & 0 & 0 \\
2 & 0 & 3 & 1 & 0 & 0 & 0 \\
3 & 0 & 1 & 2 & 0 & 0 & 0 \\
4 & 0 & 2 & 0 & 1 & 0 & 0 \\
5 & 0 & 1 & 0 & 0 & 1 & 0 \\
6 & 1 & 4 & 0 & 0 & 0 & 0 \\
7 & 1 & 2 & 1 & 0 & 0 & 0 \\
8 & 1 & 1 & 0 & 1 & 0 & 0 \\
9 & 2 & 3 & 0 & 0 & 0 & 0 \\
10 & 2 & 1 & 1 & 0 & 0 & 0 \\
11 & 3 & 2 & 0 & 0 & 0 & 0 \\
12 & 4 & 1 & 0 & 0 & 0 & 0
\end{tabular}

TABLE 2.1. Exponents of the monomials $\eta^{s} \xi_{1}^{p_{1}} \cdots \xi_{5}^{p_{5}}$ satisfying $s+p_{1}+2 p_{2}+\cdots+5 \cdot p_{5}=5$ and $p_{1} \geq 1$.

The limits corresponding to $R_{10}, R_{11}$ and $R_{12}$ do not vanish in general in dimensions $d=1$ or $d=2$; we thus shall not use these mononomials directly for the derivation of shift polynomials; however, we will employ a suitable linear combination of them below. Omitting the analogous calculation, we remark that (2.11) is also satisfied for $R_{6}=\eta \xi_{1}^{4}$ and $R_{7}=\eta \xi_{1}^{2} \xi_{2}$ in $d \geq 1$. For all the remaining monomials $R_{1}$ to $R_{5}$, property (2.11) holds trivially since these $R_{i}$ are independent of $\eta$.

Since Equation (1.3) is of sixth order, additional boundary conditions can be imposed. We choose

$$
\nabla\left(\frac{\Delta \sqrt{U}}{\sqrt{U}}\right) \cdot \nu=0 \quad \text { on } \partial \Omega .
$$

In terms of the reduction $u$, this means that we assume

$$
\frac{u_{r r r}(r)}{u(r)}+(d-1) \frac{u_{r r}(r)}{r u(r)}=0 \quad \text { at } r=1 .
$$

There are polynomials $R \in \Sigma_{5}$ for which $r^{d-1} \mathrm{D}_{R}[u, r]$ vanishes for $r \downarrow 0$ and at $r=1$ because of (2.15), and not on grounds of the homogeneous Neumann conditions alone. In analogy to the case of Neumann boundary data, these polynomials can be written in the form $R(\eta, \xi)=\left(\xi_{3}+(d-1) \xi_{2} \eta\right) Q(\eta, \xi)$ with an appropriate $Q \in \Sigma_{2}$. There is no need to consider $Q=\xi_{1}^{2}$, since then $R$ contains $\xi_{1}$ as a factor, and this has already been investigated above. It is easily seen that the choice $R=\left(\xi_{3}+(d-1) \eta \xi_{2}\right) \eta^{2}$ does not satisfy $(2.8)$ in dimension $d=1$. On the other hand, $R_{*}=\left(\xi_{3}+(d-1) \eta \xi_{2}\right) \xi_{2}$ gives

$$
\begin{aligned}
\lim _{r \downarrow 0}\left(r^{d-1} \mathrm{D}_{R_{*}}[u, r]\right) & =\lim _{r \downarrow 0} \frac{r^{d-1} u_{r r}(r) u_{r r r}(r)}{u(r)^{2}}+\left(\frac{u_{r r}(0)}{u(0)}\right)^{2} \lim _{r \downarrow 0} r^{d-2} \\
& =\frac{u_{r r}(0) u_{r r r r}(0)}{u(0)^{2}} \lim _{r \downarrow 0} r^{d}+\left(\frac{u_{r r}(0)}{u(0)}\right)^{2} \lim _{r \downarrow 0} r^{d-2} .
\end{aligned}
$$

While the first term vanishes in all dimensions $d \geq 1$, the second diverges for $d=1$ or is finite but generally nonzero for $d=2$. However, it can be annihilated by a suitable 
linear combination of $R_{10}$ and $R_{11}$. Indeed, replacing $R_{10}$ by

$$
R_{10}^{\prime}(\eta, \xi):=(d-1) \eta^{2} \xi_{1} \xi_{2}-2(d-1) \eta^{3} \xi_{1}^{2}+\left(\xi_{3}+(d-1) \eta \xi_{2}\right) \xi_{2},
$$

it is now easily verfied that $R_{10}^{\prime}$ has the property (2.8). Finally, the shift polynomial arising from the no-flux boundary condition is neglected for the same reason as in the case $K=4$ above.

In summary, we shall use the following expressions for the definition of the shift polynomials:

$$
\begin{aligned}
& R_{1}=\xi_{1}^{5}, \quad R_{2}=\xi_{1}^{3} \xi_{2}, \quad R_{3}=\xi_{1} \xi_{2}^{2}, \quad R_{4}=\xi_{1}^{2} \xi_{3}, \quad R_{5}=\xi_{1} \xi_{4}, \\
& R_{6}=\eta \xi_{1}^{4}, \quad R_{7}=\eta \xi_{1}^{2} \xi_{2}, \quad R_{8}=\eta \xi_{1} \xi_{3}, \quad R_{9}=\eta^{2} \xi_{1}^{3} \\
& R_{10}^{\prime}=\xi_{2} \xi_{3}+(d-1)\left(\eta^{2} \xi_{1} \xi_{2}-2 \eta^{3} \xi_{1}^{2}+\eta \xi_{2}^{2}\right) .
\end{aligned}
$$

The corresponding shift polynomials read as follows:

$$
\begin{aligned}
T_{1}(\eta, \xi)= & (\alpha+\beta-5) \xi_{1}^{6}+5 \xi_{1}^{4} \xi_{2}+(d-1) \eta \xi_{1}^{5} \\
T_{2}(\eta, \xi)= & (\alpha+\beta-4) \xi_{1}^{4} \xi_{2}+3 \xi_{1}^{2} \xi_{2}^{2}+\xi_{1}^{3} \xi_{3}+(d-1) \eta \xi_{1}^{3} \xi_{2} \\
T_{3}(\eta, \xi)= & (\alpha+\beta-3) \xi_{1}^{2} \xi_{2}^{2}+\xi_{2}^{3}+2 \xi_{1} \xi_{2} \xi_{3}+(d-1) \eta \xi_{1} \xi_{2}^{2} \\
T_{4}(\eta, \xi)= & (\alpha+\beta-3) \xi_{1}^{3} \xi_{3}+2 \xi_{1} \xi_{2} \xi_{3}+\xi_{1}^{2} \xi_{4}+(d-1) \eta \xi_{1}^{2} \xi_{3} \\
T_{5}(\eta, \xi)= & (\alpha+\beta-2) \xi_{1}^{2} \xi_{4}+\xi_{1} \xi_{5}+\xi_{2} \xi_{4}+(d-1) \eta \xi_{1} \xi_{4} \\
T_{6}(\eta, \xi)= & (\alpha+\beta-4) \eta \xi_{1}^{5}+4 \eta \xi_{1}^{3} \xi_{2}+(d-2) \eta^{2} \xi_{1}^{4}, \\
T_{7}(\eta, \xi)= & (\alpha+\beta-3) \eta \xi_{1}^{3} \xi_{2}+2 \eta \xi_{1} \xi_{2}^{2}+\eta \xi_{1}^{2} \xi_{3}+(d-2) \eta^{2} \xi_{1}^{2} \xi_{2}, \\
T_{8}(\eta, \xi)= & (\alpha+\beta-2) \eta \xi_{1}^{2} \xi_{3}+\eta \xi_{2} \xi_{3}+\eta \xi_{1} \xi_{4}+(d-2) \eta^{2} \xi_{1} \xi_{3} \\
T_{9}(\eta, \xi)= & (\alpha+\beta-3) \eta^{2} \xi_{1}^{4}+3 \eta^{2} \xi_{1}^{2} \xi_{2}+(d-3) \eta^{3} \xi_{1}^{3}, \\
T_{10}(\eta, \xi)= & \xi_{2} \xi_{4}+\xi_{3}^{2}+(\alpha+\beta-2) \xi_{1} \xi_{2} \xi_{3}+3(d-1) \eta \xi_{2} \xi_{3}+(d-1)(\alpha+\beta-2) \eta \xi_{1} \xi_{2}^{2} \\
& +(d-1) \eta^{2} \xi_{1} \xi_{3}+(d-1)^{2} \eta^{2} \xi_{2}^{2}+(d-1)(\alpha+\beta-2) \eta^{2} \xi_{1}^{2} \xi_{2} \\
& +(d-1)(d-7) \eta^{3} \xi_{1} \xi_{2}-2(d-1)(\alpha+\beta-2) \eta^{3} \xi_{1}^{3}-2(d-1)(d-4) \eta^{4} \xi_{1}^{2}
\end{aligned}
$$

\section{Solution of the algebraic problem}

We discuss the solution of the algebraic problem derived in the previous section and we solve two easy quantifier elimination problems.

3.1. Quantifier elimination and sum of squares. The algebraic problem stated in Lemma 2.1 is of quantifier elimination type: one is given a statement about a polynomial inequality with quantifiers over certain polynomial variables, and one wishes to find an equivalent formula in which all quantified variables are eliminated. Specifically, in (2.9), all variables except $\alpha$ are quantified, and one wants to derive a statement that involves $\alpha$ only. The latter statement provides the range of parameter value $\alpha$ such that $E_{\alpha}$ is an entropy.

Problems of this kind have been studied extensively in (real) algebraic geometry. In his pioneering work [22], Tarski has proven that a quantified formula for polynomial inequalities can be reduced to a quantifier free formula (for another set of polynomial inequalities) in an algorithmic way. He even proposed such an algorithm, which, however, is rather impractical. Nowadays, a variety of computer algebra tools are available that perform quantifier elimination efficiently. Most of them are based on cylindrical algebraic decomposition (CAD), which was originally introduced by Collins 
[6] and has since then been improved by various authors. Quantifier elimination performed by a computer with such an algorithm is equivalent to a genuine proof (to the extent to which one is willing to accept computer-aided proofs at all).

For the solution of the problems arising in the proofs of Theorems 1.1 to 1.3, we have made use of the command Reduce provided by the computer algebra software Mathematica, which uses an implementation of CAD. For Theorems 1.1 and 1.2, it has a posteriori - i.e., knowing from Mathematica's result what the solution should be been possible to write down an explicit proof, choosing suitable values for the variables $c_{i}$ and applying Lemma 3.1 and 3.2 below. For Theorem 1.3, the effort of giving an explicit proof would have been too large, so instead the output of Mathematica is presented in Appendix B.

A remark on the (im)possible extension of our method to more complicated equations is in order here. The main problem with the CAD-based algorithms is that their complexity grows doubly exponentially in the number of polynomial variables (novel algorithms with single exponential complexity, see e.g. [5], are not yet implemented). This limits the type of problems that can be dealt with in practice. The calculations involved in the computer-aided proof of Theorem 1.3 appear to be already at the edge of feasability. In fact, the solution with Mathematica was only possible after performing a priori simplifications of the problem. Entropy calculations for PDEs of order $K=8$ are currently out of reach.

An alternative - more efficient but less rigorous - approach to the solution of the specific decision problem (2.9) is provided by sum-of-squares (SOS) decompositions. Instead of verifying the existence of decision variables $c_{1}$ to $c_{m}$ for which the polynomial $S_{c}:=S_{0}+c_{1} T_{1}+\cdots+c_{m} T_{m}$ is non-negative for all $(\eta, \xi) \in \mathbb{R}^{K+1}$, one tries to determine specific values of the $c_{i}$ such that $S_{c}$ can be written as the sum of squares of polynomials in $(\eta, \xi)$. The existence of such an SOS decomposition for $S_{c}$ clearly implies its non-negativity, but it is in general far from being equivalent. The reformulation of (2.9) as an SOS problem allows for its approximate solution by application of efficient numerical optimization tools, also in situations where the complexity for CAD would be by far too high.

In contrast to the quantifier elimination algorithms discussed before, the numerical SOS method never delivers a proof of the statement, and its results will in general be sub-optimal due to the non-equivalence of positivity and the existence of a SOS decomposition. However, the SOS approach often reveals invaluable information about the suitable choice of the decision variables $c_{i}$, and this information can later be used for the simplification in the (rigorous) quantifier elimination. For a priori simplifications in the proof of Theorem 1.3, we have employed the MatLaB tool yalmip [18]; see Remark 4.1.

3.2. Two auxiliary lemmas. In this section, we solve two easy quantifier elimination problems by elementary means. These results will be useful later to perform the proofs for Theorems 1.1 and 1.2 completely explicitly, and to reduce the computational effort for proving Theorem 1.3 with computer aid.

Lemma 3.1. Let

$$
P\left(\eta, \xi_{1}, \xi_{2}\right)=a_{1} \xi_{1}^{4}+a_{2} \xi_{1}^{2} \xi_{2}+a_{3} \xi_{2}^{2}+a_{4} \eta \xi_{1}^{3}+a_{5} \eta^{2} \xi_{1}^{2}+a_{6} \eta \xi_{1} \xi_{2}
$$

be a polynomial with real coefficients. Then the quantified formula

$$
\forall\left(\eta, \xi_{1}, \xi_{2}\right) \in \mathbb{R}^{3}: P\left(\eta, \xi_{1}, \xi_{2}\right) \geq 0
$$


is equivalent to the quantifier free formula

$$
\begin{aligned}
& \text { either } a_{3}>0 \text { and } \\
& \quad\left[\left(4 a_{3} a_{5}-a_{6}^{2}>0 \text { and } 4 a_{1} a_{3} a_{5}-a_{3} a_{4}^{2}-a_{2}^{2} a_{5}-a_{1} a_{6}^{2}+a_{2} a_{4} a_{6} \geq 0\right)\right. \text { or } \\
& \left.\quad\left(4 a_{3} a_{5}-a_{6}^{2}=2 a_{4} a_{3}-a_{2} a_{6}=0 \text { and } 4 a_{3} a_{1}-a_{2}^{2} \geq 0\right)\right] \\
& \text { or } a_{3}=0 \text { and } a_{2}=a_{6}=0 \text { and } \\
& \quad\left[\left(a_{5}>0 \text { and } 4 a_{5} a_{1}-a_{4}^{2} \geq 0\right) \text { or }\left(a_{4}=a_{5}=0 \text { and } a_{1} \geq 0\right)\right] .
\end{aligned}
$$

Proof. The polynomial $P$ is nonnegative on the hyperplane $\xi_{1}=0$ if and only if $a_{3} \geq 0$. For $\xi_{1} \neq 0$, formula (3.1) is equivalent to the statement that the quadratic polynomial

$$
p\left(x_{1}, x_{2}\right)=a_{1}+a_{2} x_{2}+a_{3} x_{2}^{2}+a_{4} x_{1}+a_{5} x_{1}^{2}+a_{6} x_{1} x_{2}
$$

is nonnegative for all real values $x_{1}=\eta / \xi_{1}$ and $x_{2}=\xi_{2} / \xi_{1}^{2}$. For fixed $x_{1}^{*} \in \mathbb{R}$, the quadratic polynomial in $x_{2}$,

$$
p\left(x_{1}^{*}, x_{2}\right)=\left(a_{1}+a_{4} x_{1}^{*}+a_{5}\left(x_{1}^{*}\right)^{2}\right)+\left(a_{2}+a_{6} x_{1}^{*}\right) x_{2}+a_{3} x_{2}^{2},
$$

is nonnegative if and only if

$$
\begin{aligned}
& \text { either } a_{3}>0 \text { and } q_{1}\left(x_{1}^{*}\right):=4 a_{3}\left(a_{1}+a_{4} x_{1}^{*}+a_{5}\left(x_{1}^{*}\right)^{2}\right)-\left(a_{2}+a_{6} x_{1}^{*}\right)^{2} \geq 0 \\
& \text { or } a_{3}=0 \text { and } q_{2}\left(x_{1}^{*}\right):=a_{2}+a_{6} x_{1}^{*}=0 \text { and } q_{3}\left(x_{1}^{*}\right):=a_{1}+a_{4} x_{1}^{*}+a_{5}\left(x_{1}^{*}\right)^{2} \geq 0 .
\end{aligned}
$$

Therefore, $p\left(x_{1}, x_{2}\right)$ is nonnegative if and only if $q_{1}\left(x_{1}\right) \geq 0$ or if $q_{2}\left(x_{1}\right)=0$ and $q_{3}\left(x_{1}\right) \geq$ 0 for all $x_{1} \in \mathbb{R}$. The polynomial

$$
q_{1}\left(x_{1}\right)=4 a_{3} a_{1}-a_{2}^{2}+2\left(2 a_{3} a_{4}-a_{2} a_{6}\right) x_{1}+\left(4 a_{3} a_{5}-a_{6}^{2}\right) x_{1}^{2}
$$

is nonnegative if and only if

$$
\begin{aligned}
& \text { either } 4 a_{3} a_{5}-a_{6}^{2}>0 \text { and }\left(4 a_{3} a_{5}-a_{6}^{2}\right)\left(4 a_{3} a_{1}-a_{2}^{2}\right)-\left(2 a_{3} a_{4}-a_{2} a_{6}\right)^{2} \geq 0 \\
& \text { or } 4 a_{3} a_{5}-a_{6}^{2}=2 a_{4} a_{3}-a_{2} a_{6}=0 \text { and } 4 a_{3} a_{1}-a_{2}^{2} \geq 0 .
\end{aligned}
$$

The polynomial $q_{2}$ vanishes on $\mathbb{R}$ if and only if $a_{2}=a_{6}=0$, and $q_{3}\left(x_{1}\right)=a_{1}+a_{4} x_{1}+$ $a_{5} x_{1}^{2}$ is nonnegative if and only if

$$
\begin{aligned}
& \text { either } a_{5}>0 \text { and } 4 a_{5} a_{1}-a_{4}^{2} \geq 0 \\
& \text { or } a_{4}=a_{5}=0 \text { and } a_{1} \geq 0 .
\end{aligned}
$$

Inserting these statements into (3.3) yields (3.2).

Lemma 3.2. Let the polynomial $P(x)=b_{0}+b_{1} x+b_{2} x^{2}$ with $b_{2} \geq 0$ and real numbers $z_{1}<z_{2}$ be given. Then the quantified formula

$$
\exists x \in\left(z_{1}, z_{2}\right): P(x) \leq 0
$$

is equivalent to the quantifier free expression

$$
\begin{aligned}
& \text { either } b_{2}>0 \text { and }\left[b_{0}+b_{1} z_{1}+b_{2} z_{1}^{2}<0 \text { or }\left(4 b_{0} b_{2}-b_{1}^{2} \leq 0 \text { and } 2 b_{2} z_{1}+b_{1}<0\right)\right] \\
& \quad \text { and }\left[b_{0}+b_{1} z_{2}+b_{2} z_{2}^{2}<0 \text { or }\left(4 b_{0} b_{2}-b_{1}^{2} \leq 0 \text { and } 2 b_{2} z_{2}+b_{1}>0\right)\right] \\
& \text { or } b_{2}=0 \text { and }\left[\left(b_{1}>0 \text { and } b_{0}+b_{1} z_{1}<0\right) \text { or }\left(b_{1}<0 \text { and } b_{0}+b_{1} z_{2}<0\right)\right. \\
& \left.\quad \text { or }\left(b_{1}=0 \text { and } b_{0} \leq 0\right)\right] \text {. }
\end{aligned}
$$


Proof. First assume that $b_{2}>0$. Then the quadratic polynomial $P$ is nonpositive in some interval if and only if $4 b_{0} b_{2}-b_{1}^{2} \leq 0$ and exactly for those $x$ which lie in between the two real roots $x_{ \pm}=\left( \pm \sqrt{b_{1}^{2}-4 b_{0} b_{2}}-b_{1}\right) / 2 b_{2}$. The statement (3.4) is then equivalent to $z_{1}<x_{+}$and $z_{2}>x_{-}$, which can be rephrased as the first two lines of (3.5). Indeed, if $z_{1}+b_{1} / 2 b_{2}<0$ then $z_{1}<x_{+}$is always satisfied, and if $z_{1}+b_{1} / 2 b_{2} \geq 0$ then $z_{1}<x_{+}$is equivalent to $b_{0}+b_{1} z_{1}+b_{2} z_{1}^{2}<0$. Notice that this inequality is satisfied only if $4 b_{0} b_{2}-b_{1}^{2} \leq 0$.

If $b_{2}=0$, then $P$ is linear. If additionally $b_{1}=0,(3.4)$ is equivalent to $b_{0} \leq 0$. Therefore, let $b_{1} \neq 0$. Then $P$ vanishes at $x_{0}=-b_{0} / b_{1}$, and (3.4) is equivalent to $z_{1}<x_{0}$ (if $b_{1}>0$ ) or $z_{2}>x_{0}$ (if $b_{1}<0$ ). This leads to the last two lines of (3.5).

\section{Proofs of the theorems}

4.1. Proof of Theorem 1.1. By Example 2.1 (A) and (2.6), the canonical symbol of (1.1) reads as follows:

$$
S_{0}(\eta, \xi)=-\xi_{1} \xi_{3}-(d-1) \eta \xi_{1} \xi_{2}+(d-1) \eta^{2} \xi_{1}^{2} .
$$

We have to solve the decision problem

$$
\exists c_{1}, c_{2}, c_{3} \in \mathbb{R}: \forall\left(\eta, \xi_{1}, \xi_{2}, \xi_{3}\right) \in \mathbb{R}^{4}: S(\eta, \xi)=\left(S_{0}+c_{1} T_{1}+c_{2} T_{2}+c_{3} T_{3}\right)(\eta, \xi) \geq 0,
$$

where the shift polynomials $T_{1}, T_{2}$, and $T_{3}$ are given by (2.12)-(2.14).

This problem can be simplified. Indeed, the variable $\xi_{3}$ appears in $S$ only in the term $\xi_{1} \xi_{3}$, and its coefficient $-1+c_{1}$ has to vanish; otherwise, $S(\eta, \xi)$ would become negative for $\xi_{1} \equiv 1$ and $\xi_{3} \rightarrow \pm \infty$ if $c_{1} \lessgtr 1$. Thus, $c_{1}=1$, and the decision problem reduces to finding $c_{2}, c_{3} \in \mathbb{R}$ such that for all $(\eta, \xi)=\left(\eta, \xi_{1}, \xi_{2}\right) \in \mathbb{R}^{3}$,

$$
\begin{aligned}
S(\eta, \xi) & =\left(S_{0}+T_{1}+c_{2} T_{2}+c_{3} T_{3}\right)(\eta, \xi) \\
& =a_{1} \xi_{1}^{4}+a_{2} \xi_{1}^{2} \xi_{2}+a_{3} \xi_{2}^{2}+a_{4} \eta \xi_{1}^{3}+a_{5} \eta^{2} \xi_{1}^{2}+a_{6} \eta \xi_{1} \xi_{2} \geq 0,
\end{aligned}
$$

where, setting $\gamma=\alpha+\beta$,

$$
\begin{array}{lll}
a_{1}=(\gamma-3) c_{2}, & a_{2}=\gamma-2+3 c_{2}, & a_{3}=1, \\
a_{4}=(\gamma-2) c_{3}+(d-1) c_{2}, & a_{5}=(d-2) c_{3}+d-1, & a_{6}=2 c_{3} .
\end{array}
$$

In this proof, we perform the quantifier elimination explicitly, without computer aid. By Lemma 3.1, this decision problem is equivalent to either

$$
\begin{aligned}
& 0<4 a_{3} a_{5}-a_{6}^{2}=-4\left(c_{3}+1\right)\left(c_{3}-d+1\right)=:-4 C, \\
& \begin{aligned}
0 \leq q\left(c_{2}, c_{3}\right): & =4 a_{1} a_{3} a_{5}-a_{3} a_{4}^{2}-a_{2}^{2} a_{5}-a_{1} a_{6}^{2}+a_{2} a_{4} a_{6} \\
& =\left(9 C-\left(d-3 c_{3}-1\right)^{2}\right) c_{2}^{2}+2 C \gamma c_{2}+(\gamma-2)^{2} C
\end{aligned}
\end{aligned}
$$

or

$$
\begin{aligned}
0 & =4 a_{3} a_{5}-a_{6}^{2}=-4\left(c_{3}+1\right)\left(c_{3}-d+1\right) \\
0 & =2 a_{3} a_{4}-a_{2} a_{6}=2 c_{2}\left(d-3 c_{3}-1\right) \\
0 & \leq 4 a_{1} a_{3}-a_{2}^{2}=4(\gamma-3) c_{2}-\left(3 c_{2}+\gamma-2\right)^{2} \\
& =-9\left(c_{2}+\frac{\gamma}{9}\right)^{2}+\frac{8}{9}(3-\gamma)\left(\gamma-\frac{3}{2}\right)
\end{aligned}
$$

First, we solve (4.4)-(4.6). Equation (4.5) yields $c_{2}=0$ or $c_{3}=(d-1) / 3$. Because of (4.4), the latter case is only possible if $d=1$. Let $c_{2}=0$. Then (4.6) is fulfilled if and 
only if $\gamma=2$. On the other hand, if $c_{3}=(d-1) / 3$ (and hence, $d=1$ ), the largest range for $\gamma$ fulfilling (4.6) is obtained by choosing the maximizing value $c_{2}=-\gamma / 9$. With this choice, (4.6) is fulfilled if and only if $3 / 2 \leq \gamma \leq 3$. This shows that (4.4)-(4.6) holds for some $c_{2}, c_{3} \in \mathbb{R}$ if and only if $d=1$ and $3 / 2 \leq \gamma \leq 3$ or if $d>1$ and $\gamma=2$.

Next, we solve (4.2)-(4.3). The first inequality implies that $-1<c_{3}<d-1$. For any fixed $c_{3}$, the polynomial $q\left(c_{2}, c_{3}\right)$ is quadratic in $c_{2}$ with a strictly negative leading coefficient (since $C<0$ by (4.2)). Thus, there exists $c_{2} \in \mathbb{R}$ such that $q\left(c_{2}, c_{3}\right) \leq 0$ if and only if the discriminant of $q\left(\cdot, c_{3}\right)$ is nonnegative:

$$
0 \leq(2 C \gamma)^{2}-4\left(9 C-\left(d-3 c_{3}-1\right)^{2}\right)(\gamma-2)^{2} C=4 C \Delta\left(c_{3}\right)
$$

where

$$
\Delta\left(c_{3}\right)=\gamma^{2} c_{3}^{2}+3(\gamma-2)^{2}\left(d-4-\gamma^{2} d\right) c_{3}+(\gamma-2)^{2}(d-1)(d+8)+\gamma^{2}-\gamma^{2} d .
$$

Since $C<0$, the discriminant is nonnegative if and only if the quadratic polynomial $\Delta\left(c_{3}\right)$ is nonpositive for some $-1<c_{3}<d-1$. By Lemma 3.2, this is the case if either $d=1$ and $3 / 2<\gamma<3$ or $d>1$ and $3 / 2 \leq \gamma \leq 3$. Thus, there exist $c_{2} \in \mathbb{R}, c_{3} \in(-1, d-1)$ such that (4.2)-(4.3) holds if and only if $3 / 2 \leq \gamma \leq 3$. This shows that $E_{\alpha}$ are entropies for all $3 / 2 \leq \alpha+\beta \leq 3$.

We wish to quantify the constant $c>0$ in the entropy production inequality (1.4) for the choice

$$
Q_{\alpha}[U]=\int_{\Omega}\left(\Delta U^{\gamma / 2}\right)^{2} \mathrm{~d} x=\omega_{d} \int_{0}^{1} u^{\gamma} \mathrm{D}_{W}[u, r] r^{d-1} \mathrm{~d} r .
$$

The symbol $W$ that characterizes $Q_{\alpha}$ is

$$
\begin{aligned}
W(\eta, \xi)= & \left(\frac{\gamma}{2}\right)^{2}\left(\frac{\gamma}{2}-1\right)^{2} \xi_{1}^{4}+2\left(\frac{\gamma}{2}\right)^{2}\left(\frac{\gamma}{2}-1\right) \xi_{1}^{2} \xi_{2}+\left(\frac{\gamma}{2}\right)^{2} \xi_{2}^{2} \\
& +2(d-1)\left(\frac{\gamma}{2}\right)^{2}\left(\frac{\gamma}{2}-1\right) \eta \xi_{1}^{3}+(d-1)^{2}\left(\frac{\gamma}{2}\right)^{2} \eta^{2} \xi_{1}^{2}+2(d-1)\left(\frac{\gamma}{2}\right)^{2} \eta \xi_{1} \xi_{2} .
\end{aligned}
$$

We wish to find the largest $c>0$ for which there exist $c_{2}, c_{3} \in \mathbb{R}$ such that for all $(\eta, \xi)=\left(\eta, \xi_{1}, \xi_{2}\right) \in \mathbb{R}^{3}$,

$$
S_{c}(\eta, \xi)=(S-c W)(\eta, \xi)=a_{1} \xi_{1}^{4}+a_{2} \xi_{1}^{2} \xi_{2}+a_{3} \xi_{2}^{2}+a_{4} \eta \xi_{1}^{3}+a_{5} \eta^{2} \xi_{1}^{2}+a_{6} \eta \xi_{1} \xi_{2} \geq 0,
$$

where

$$
\begin{aligned}
& a_{1}=(\gamma-3) c_{2}-c\left(\frac{\gamma}{2}\right)^{2}\left(\frac{\gamma}{2}-1\right)^{2}, \\
& a_{2}=\gamma-2+3 c_{2}-2 c\left(\frac{\gamma}{2}\right)^{2}\left(\frac{\gamma}{2}-1\right) \\
& a_{3}=1-c\left(\frac{\gamma}{2}\right)^{2} \\
& a_{4}=(\gamma-2) c_{3}+(d-1) c_{2}-2 c(d-1)\left(\frac{\gamma}{2}\right)^{2}\left(\frac{\gamma}{2}-1\right), \\
& a_{5}=(d-2) c_{3}+d-1-c(d-1)^{2}\left(\frac{\gamma}{2}\right)^{2} \\
& a_{6}=2 c_{3}-2 c(d-1)\left(\frac{\gamma}{2}\right)^{2}
\end{aligned}
$$


We consider the cases $a_{3}>0$ and $a_{3}=0$ separately. First, let $a_{3}=0$, which is equivalent to $c=4 / \gamma^{2}$. By Lemma 3.1, we find that $a_{2}=a_{6}=0$, which gives $c_{2}=0$ and $c_{3}=d-1$. Furthermore, we obtain $a_{5}=0$. Hence, by the same lemma, $a_{4}=0$ and $a_{1}=-(\gamma / 2-$ $1)^{2} \geq 0$, implying that $\gamma=2$. Next, let $a_{3}>0$. By Lemma 3.1, the nonnegativity of $S_{c}$ for certain values $c, c_{2}$, and $c_{3}$ is equivalent to either

$$
\begin{aligned}
0 & <4 a_{3} a_{5}-a_{6}^{2}=-\left(c_{3}-d+1\right)\left(4 c_{3}-\gamma^{2} d c+4\right)=:-E \\
0 & \leq q\left(c_{2}, c_{3}, c\right):=4 a_{1} a_{3} a_{5}-a_{3} a_{4}^{2}-a_{2}^{2} a_{5}-a_{1} a_{6}^{2}+a_{2} a_{4} a_{6} \\
& =\frac{1}{4-\gamma^{2} c}\left(9 E-\left(2 d-2-6 c_{3}+\gamma^{2}(d-1)\right)^{2} c\right) c_{2}^{2}+\frac{E}{2} \gamma c_{2}+\frac{E}{4}(\gamma-2)^{2}
\end{aligned}
$$

or

$$
\begin{aligned}
0 & =4 a_{3} a_{5}-a_{6}^{2}=-\left(c_{3}-d+1\right)\left(4 c_{3}-\gamma^{2} c d+4\right), \\
0 & =2 a_{3} a_{4}-a_{2} a_{6}=c_{2}\left(2 d-2-6 c_{3}+\gamma^{2} c(d-1)\right), \\
0 & \leq 4 a_{1} a_{3}-a_{2}^{2}=-9 c_{2}^{2}+\frac{\gamma}{2}\left(\gamma^{2} c-4\right)+\frac{1}{2}(\gamma-2)^{2}\left(\gamma^{2} c-4\right) \\
& =-9\left(c_{2}-\frac{\gamma}{36}\left(\gamma^{2} c-4\right)\right)^{2}+\frac{1}{144}\left(\gamma^{2} c-4\right)\left(\gamma^{4} c+32 \gamma^{2}+144(1-\gamma)\right) .
\end{aligned}
$$

First, we solve (4.9)-(4.11). We obtain a maximal value for $c$ by choosing $c_{2}=\gamma\left(\gamma^{2} c-\right.$ $4) / 36$. Since $a_{3}=1-\gamma^{2} c / 4>0$ by assumption, we have $c_{2}<0$. With this choice of $c_{2}$, condition (4.11) implies that $c \leq 16(2 \gamma-3)(3-\gamma) / \gamma^{4}$. Furthermore, by (4.10), $c_{3}=(d-1)\left(\gamma^{2} c+2\right) / 6$. Condition (4.9) can be satisfied only if $d=1$.

Next, we consider (4.7)-(4.8). The polynomial $q\left(\cdot, c_{3}, c\right)$ is quadratic in $c_{2}$ with a negative leading coefficient (since $a_{3}>0$ ). Hence, there exists $c_{2} \in \mathbb{R}$ such that $q\left(c_{2}, c_{3}, c\right)$ is nonnegative if and only if its discriminant $D\left(c_{3}, c\right)=E \Delta_{0}\left(c_{3}, c\right) / 4$ is nonnegative, where $E<0$ (by (4.7)) and

$$
\begin{aligned}
\Delta_{0}\left(c_{3}, c\right)= & 4 \gamma^{2} c_{3}^{2}+\left(8 \gamma^{2}+12(\gamma-2)^{2}(d-4)-4 \gamma^{2} d-\gamma^{4} c d\right) c_{3} \\
& +4(\gamma-2)^{2}(d-1)(d+8)-4 \gamma^{2} d+4 \gamma^{2}-4 \gamma^{2} c(\gamma-2)^{2}(d-1)^{2}-\gamma^{4} c d+\gamma^{4} c d^{2}
\end{aligned}
$$

is a quadratic polynomial in $c_{3}$. Applying Lemma 3.2, we find that

$$
\begin{array}{ll}
\text { if } d=1 \text { and } \gamma \in\left(\frac{3}{2}, 3\right): & c<\frac{16}{\gamma^{4}}(2 \gamma-3)(3-\gamma) ; \\
\text { if } d>1 \text { and } \gamma \in\left(\frac{3}{2}, 3\right) \backslash\{2\}: & c \leq \frac{16}{\gamma^{4}}(2 \gamma-3)(3-\gamma) .
\end{array}
$$

The case $a_{3}=0$ provides the choice $\gamma=2$ with $c=16 / \gamma^{4}=1$. This proves the theorem.

4.2. Proof of Theorem 1.2. By Example 2.1 (B), the canonical symbol $S_{0}$ for entropy dissipation along the DLSS Equation (1.2) is given by

$$
S_{0}(\eta, \xi)=-\frac{1}{2} \xi_{1} \xi_{3}+\xi_{2} \xi_{1}^{2}-\frac{1}{2} \xi_{1}^{4}-\frac{1}{2}(d-1) \eta \xi_{1}\left(\xi_{2}-\xi_{1}^{2}-\eta \xi_{1}\right) .
$$

Again, we have to solve the decision problem (4.1). The same argument as in the previous subsection shows that $c_{1}=1$. Thus, we wish to find $c_{2}, c_{3} \in \mathbb{R}$ such that for all $(\eta, \xi)=\left(\eta, \xi_{1}, \xi_{2}\right) \in \mathbb{R}^{3}$,

$$
2 S(\eta, \xi)=a_{1} \xi_{1}^{4}+a_{2} \xi_{1}^{2} \xi_{2}+a_{3} \xi_{2}^{2}+a_{4} \eta \xi_{1}^{3}+a_{5} \eta^{2} \xi_{1}^{2}+a_{6} \eta \xi_{1} \xi_{2} \geq 0,
$$

where 


$$
\begin{array}{lll}
a_{1}=(\alpha-3) c_{2}-1, & a_{2}=\alpha+3 c_{2}, & a_{3}=1, \\
a_{4}=(\alpha-2) c_{3}+(d-1)\left(c_{2}+1\right), & a_{5}=(d-2) c_{3}+d-1, & a_{6}=2 c_{3} .
\end{array}
$$

According to Lemma 3.1, the above decision problem is equivalent to either

$$
\begin{aligned}
0<4 a_{3} a_{5}-a_{6}^{2}= & -4\left(c_{3}+1\right)\left(c_{3}-d+1\right)=:-4 C \\
0 \leq q\left(c_{2}, c_{3}\right):= & 4 a_{1} a_{3} a_{5}-a_{3} a_{4}^{2}-a_{2}^{2} a_{5}-a_{1} a_{6}^{2}+a_{2} a_{4} a_{6} \\
= & \left(9 C-\left(d-3 c_{3}-1\right)^{2}\right) c_{2}^{2}-2\left(d^{2}+4 d+(d-7) c_{3}-5-\alpha C\right) c_{2} \\
& +\alpha^{2} C-d^{2}-2 d+4 c_{3}+3
\end{aligned}
$$

or

$$
\begin{aligned}
& 0=4 a_{3} a_{5}-a_{6}^{2}=-4\left(c_{3}+1\right)\left(c_{3}-d+1\right), \\
& 0=2 a_{3} a_{4}-a_{2} a_{6}=-2\left(c_{2}+2 c_{3}+3 c_{2} c_{3}+1\right)+2\left(c_{2}+1\right) d, \\
& 0 \leq 4 a_{1} a_{3}-a_{2}^{2}=-4-\alpha^{2}-12 c_{2}-2 \alpha c_{2}-9 c_{2}^{2} .
\end{aligned}
$$

First, we solve (4.14)-(4.16). Condition (4.14) implies that either $c_{3}=-1$ or $c_{3}=d-1$. In the former case, $(4.15)$ gives $c_{2}=-(d+1) /(d+2)$. Then (4.16) is equivalent to

$$
\alpha^{2}-\frac{2(d+1)}{d+2} \alpha+\frac{(d-1)^{2}}{2(d+2)^{2}} \leq 0
$$

which is satisfied if and only if

$$
\frac{(\sqrt{d}-1)^{2}}{d+2} \leq \alpha \leq \frac{(\sqrt{d}+1)^{2}}{d+2} .
$$

In the latter case $c_{3}=d-1,(4.15)$ is satisfied if $d=1$ or if $d>1$ and $c_{2}=-1 / 2$. If $d=1$, we choose the maximizing value $c_{2}=-(\alpha+6) / 9$ for (4.16). Then, this inequality is satisfied if and only if $0 \leq \alpha \leq 3 / 2$. On the other hand, if $d>1$, (4.16) can be written as $\alpha^{2}-\alpha+1 / 4 \leq 0$, which is satisfied if and only if $\alpha=1 / 2$. We have shown that the decision problem is solvable if $d=1$ and $0 \leq \alpha \leq 3 / 2$ or if $d>1$ and (4.17) hold.

Next, we solve (4.12)-(4.13). The discriminant $D\left(c_{3}\right)$ of the quadratic polynomial $q\left(\cdot, c_{3}\right)$ factorizes as $D\left(c_{3}\right)=4 C \Delta\left(c_{3}\right)$, where

$$
\begin{aligned}
\Delta\left(c_{3}\right)= & \alpha^{2} c_{3}^{2}+2\left(\alpha^{2}(d-5)-\alpha(d-7)\right) c_{3}+\left(d^{2}+6 d-7\right) \alpha^{2} \\
& -2 \alpha\left(d^{2}+4 d-5\right)+(d-1)^{2} .
\end{aligned}
$$

Notice that $C<0$ by (4.12). An application of Lemma 3.2 shows that $D\left(c_{3}\right)$ is nonnegative if $d=1$ and $0<\alpha<3 / 2$, or $d \in\{2,3\}$ and $(\sqrt{d}-1)^{2} /(d+2)<\alpha \leq 3 / 2$, or $d \in$ $\{4,5,6,7\}$ and $(\sqrt{d}-1)^{2} /(d+2)<\alpha<(\sqrt{d}+1)^{2} /(d+2)$, or $d \geq 8$ and $(d-4) /(2 d-4) \leq$ $\alpha<(\sqrt{d}+1)^{2} /(d+2)$. This proves that $d E_{\alpha} / d t \leq 0$ if these conditions are satisfied.

The estimates for the entropy production term $\omega_{d} \int\left(\Delta_{r} u^{\alpha / 2}\right)^{2} r^{d-1} \mathrm{~d} r$ are obtained by similar arguments as in the previous subsection. We leave the details to the reader.

4.3. Proof of Theorem 1.3. The canonical symbol associated to the sixthorder Equation (1.3) can be read off from the representation of its radially symmetric solutions as given in Example $2.1(\mathrm{C})$. One finds

$$
\begin{aligned}
S_{0}(\eta, \xi)= & 6 \xi_{1}^{6}-18 \xi_{1}^{4} \xi_{2}+11 \xi_{1}^{2} \xi_{2}^{2}+8 \xi_{1}^{3} \xi_{3}-3 \xi_{1}^{2} \xi_{4}-5 \xi_{1} \xi_{2} \xi_{3}+\xi_{1} \xi_{5} \\
& +(d-1)\left[-6 \eta \xi_{1}^{5}+(2 d-7) \eta^{2} \xi_{1}^{4}+14 \eta \xi_{1}^{3} \xi_{2}+(3 d-8) \eta^{3} \xi_{1}^{3}-4 \eta \xi_{1} \xi_{2}^{2}\right. \\
& -3(d-4) \eta^{2} \xi_{1}^{2} \xi_{2}-6 \eta \xi_{1}^{2} \xi_{3}+3(d-3) \eta^{4} \xi_{1}^{2}-3(d-3) \eta^{3} \xi_{1} \xi_{2}+(d-5) \eta^{2} \xi_{1} \xi_{3} \\
& \left.+2 \eta \xi_{1} \xi_{4}\right] .
\end{aligned}
$$


We have to solve the decision problem

$$
\exists c_{1}, \ldots, c_{10} \in \mathbb{R}: \forall(\eta, \xi): S(\eta, \xi)=\left(S_{0}+c_{1} T_{1}+\cdots+c_{10} T_{10}\right)(\eta, \xi) \geq 0,
$$

where the shift polynomials $T_{i}$ are given by (2.16)-(2.25) with $\beta=0$. Again, we can simplify this problem by eliminating the terms whose sign cannot be controlled. We choose $c_{3}=0$ to eliminate $\xi_{2}^{3}, c_{5}=-1$ to eliminate $\xi_{1} \xi_{5}, c_{8}=-(d-1)$ to eliminate $\eta \xi_{1} \xi_{4}, c_{4}=\alpha-2$ to eliminate $\xi_{1}^{2} \xi_{4}$, and $c_{10}=1$ to eliminate the product $\xi_{2} \xi_{4}$ introduced by $T_{5}$. With these choices,

$$
\begin{aligned}
S(\eta, \xi)= & \left(c_{1} T_{1}+c_{2} T_{2}+0 \cdot T_{3}+(\alpha-2) T_{4}+(-1) \cdot T_{5}+c_{6} T_{6}+c_{7} T_{7}-(d-1) T_{8}\right. \\
& \left.+c_{9} T_{9}+1 \cdot T_{10}\right)(\eta, \xi) \\
= & \left((\alpha-5) c_{1}+6\right) \xi_{1}^{6}+\left(5 c_{1}+(\alpha-4) c_{2}-18\right) \xi_{1}^{4} \xi_{2}+\left(3 c_{2}+11\right) \xi_{1}^{2} \xi_{2}^{2} \\
& +\left(c_{2}+(\alpha+1)(\alpha-3)+8\right) \xi_{1}^{3} \xi_{3}+(3 \alpha-5) \xi_{1} \xi_{2} \xi_{3} \\
& +\left((\alpha-4) c_{6}+(d-1)\left(c_{1}-6\right)\right) \eta \xi_{1}^{5} \\
& +\left((\alpha-3) c_{9}+(d-2) c_{6}+(d-1)(2 d-7)\right) \eta^{2} \xi_{1}^{4} \\
& +\left((\alpha-3) c_{7}+4 c_{6}+(d-1)\left(c_{2}+14\right)\right) \eta \xi_{1}^{3} \xi_{2} \\
& +\left((d-3) c_{9}-2(\alpha-2)(d-1)+(d-1)(3 d-8)\right) \eta^{3} \xi_{1}^{3} \\
& +\left(2 c_{7}+(\alpha-6)(d-1)\right) \eta \xi_{1} \xi_{2}^{2} \\
& +\left((\alpha-2)(d-1)+3 c_{9}+(d-2) c_{7}-3(d-1)(d-4)\right) \eta^{2} \xi_{1}^{2} \xi_{2} \\
& +\left(c_{7}-3(d-1)\right) \eta \xi_{1}^{2} \xi_{3}+(d-1)^{2} \eta^{4} \xi_{1}^{2}-2(d-1)^{2} \eta^{3} \xi_{1} \xi_{2} \\
& -2(d-1) \eta^{2} \xi_{1} \xi_{3}+2(d-1) \eta \xi_{2} \xi_{3}+(d-1)^{2} \eta^{2} \xi_{2}^{2}+\xi_{3}^{2} .
\end{aligned}
$$

The corresponding decision problem contains the four variables $\eta, \xi_{1}, \ldots, \xi_{3}$ and the five coefficients $c_{1}, c_{2}, c_{6}, c_{7}$ and $c_{9}$. For further simplification, we make a change of variables. Let

$$
\zeta_{1}=\frac{\eta}{\xi_{1}}, \quad \zeta_{2}=\frac{\xi_{2}}{\xi_{1}^{2}}-\frac{\eta}{\xi_{1}}, \quad \zeta_{3}=\frac{\xi_{3}}{\xi_{1}^{3}}-3 \frac{\eta}{\xi_{1}}\left(\frac{\xi_{2}}{\xi_{1}^{2}}-\frac{\eta}{\xi_{1}}\right) .
$$

These definitions are motivated by the observation that for any radially symmetric function $U(x)=u(r)$, the tensors $\nabla_{x} U, \nabla_{x}^{2} U$ and $\nabla_{x}^{3} U$ of the first, second and third total derivatives take the form

$$
\begin{aligned}
& \nabla_{x} U(x)=u \xi_{1} \mathbf{e}_{r}, \\
& \nabla_{x}^{2} U(x)=u \xi_{1}^{2}\left(\zeta_{2} \mathbf{e}_{r} \otimes \mathbf{e}_{r}+\zeta_{1} \mathbf{1}\right) \\
& \nabla_{x}^{3} U(x)=u \xi_{1}^{3}\left(\zeta_{3} \mathbf{e}_{r} \otimes \mathbf{e}_{r} \otimes \mathbf{e}_{r}+\zeta_{1} \zeta_{2} \mathbf{e}_{r} \otimes_{s} \mathbf{1}\right),
\end{aligned}
$$

where $\left(\mathbf{e}_{r} \otimes_{s} \mathbf{1}\right)_{i j k}=\delta_{i j} x_{k}+\delta_{j k} x_{i}+\delta_{i k} x_{j}$. It turns out that $S$ can be expressed in terms of $\zeta=\left(\zeta_{1}, \zeta_{2}, \zeta_{3}\right)$ only. Furthermore, choosing $c_{7}=-c_{9}=(\alpha+1 / 2)(d-1)-$ see Remark 4.1 below - some higher-order terms cancel, and we end up with $S_{1}(\zeta)=$ $\xi_{1}^{6} S(\eta, \xi)$, which is defined in Appendix B in input line 6 of the Mathematica notebook. For any fixed $\zeta_{1}$ and $\zeta_{2}$, the polynomial $S_{1}(\zeta)$ is quadratic in $\zeta_{3}$, with leading coefficient equal to one. This quadratic polynomial is nonnegative if and only if its discriminant

$$
D\left(\zeta_{1}, \zeta_{2}\right)=\left(\partial_{\zeta_{3}} S_{1}\left(\zeta_{1}, \zeta_{2}, 0\right)\right)^{2}-4 S_{1}\left(\zeta_{1}, \zeta_{2}, 0\right)
$$


is nonpositive. Thus, the nonnegativity of $S_{0}$ for some coefficients $c_{i}$ is reduced to the following decision problem:

$$
\exists c_{1}, c_{2}, c_{6} \in \mathbb{R}: \forall \zeta_{1}, \zeta_{2} \in \mathbb{R}:-D\left(\zeta_{1}, \zeta_{2}\right) \geq 0 .
$$

The discriminant $D\left(\zeta_{1}, \zeta_{2}\right)$ is again of quadratic type, now in terms of $\zeta_{1}$ and $\zeta_{2}$. Thus Lemma 3.1 is applicable and yields several conditions on $c_{1}, c_{2}$, and $c_{6}$ for the nonpositivity of $D$. This nonlinear system of equations and inequalities is solved by the computer algebra system Mathematica (see Appendix B for more details). As a result, we obtain, for given dimension $d \geq 1$, conditions on the admissible values of $\alpha$. More precisely, $\alpha$ has to be in between the numbers $\alpha_{0}(d)$ and $\alpha_{1}(d)$, and $\alpha_{i}(d)$ are the positive roots of certain higher-order polynomials which are explicit. Their roots, however, can be calculated only numerically and are given in the statement of the theorem.

The entropy production

$$
\omega_{d} \int_{0}^{1}\left(\left(\Delta_{r} \sqrt{u}\right)_{r}^{2}+(\sqrt[6]{u})_{r}^{6}\right) r^{d-1} \mathrm{~d} r
$$

is represented by the symbol

$$
\begin{aligned}
W(\eta, \xi) & =\frac{1}{4}\left(\xi_{3}-\frac{3}{2} \xi_{2} \xi_{1}+\frac{3}{4} \xi_{1}^{3}+(d-1)\left(\eta \xi_{2}-\frac{1}{2} \eta \xi_{1}^{2}-\eta^{2} \xi_{1}\right)\right)^{2}+\frac{1}{46656} \xi_{1}^{6} \\
& =\frac{\xi_{1}^{6}}{64}\left(4 \zeta_{3}+\left(2 \zeta_{2}-1\right)\left((2 d+4) \zeta_{1}-3\right)\right)^{2}+\frac{\xi_{1}^{6}}{46656}=: \xi_{1}^{6} W_{1}(\zeta) .
\end{aligned}
$$

Setting $\alpha=1$ in the definition of $S_{1}(\zeta)$, we obtain the decision problem

$$
\exists c_{1}, c_{2}, c_{6} \in \mathbb{R}, c>0: \forall \zeta=\left(\zeta_{1}, \zeta_{2}, \zeta_{3}\right) \in \mathbb{R}^{3}: S_{1}(\zeta)-c W_{1}(\zeta) \geq 0
$$

Our solution strategy is the same as before. We observe that $S_{1}-c W_{1}$ is a quadratic polynomial in $\zeta_{3}$, and we calculate the respective discriminant. The latter turns out to be quadratic in the remaining variables $\zeta_{1}$ and $\zeta_{2}$. Omitting the details, we remark that the reduced decision problem for the discriminant is again solvable with the aid of Lemma 3.1 and Mathematica. This results in numerical values for $c>0$ such that (1.4) holds.

REMARK 4.1. The ad hoc choice of the coefficients $c_{7}$ and $c_{9}$ in the proof was originally motivated by the numerical result for the SOS decomposition of $S_{1}$ obtained with yalmip [18]. There are several reasons to believe that this choice is indeed optimal: First, $c_{9}=-c_{7}$ cancels the coefficient of the indefinite term $\zeta_{1}^{3}$, which is obtained after rewriting $S_{1}$ in terms of $\left(\zeta_{1}, \zeta_{2}, \zeta_{3}\right)$. Second, with $c_{7}=(\alpha+1 / 2)(d-1)$, the coefficient of the term $\zeta_{1} \zeta_{2}^{2}$ in the discriminant $D\left(\zeta_{1}, \zeta_{2}\right)$ vanishes, such that the remaining polynomial becomes quadratic in $\zeta_{1}$ and $\zeta_{2}$.

\section{Absence of entropies}

Similarly as in $[11,17]$, it is possible to prove that certain functionals $E_{\alpha}$ cannot be entropies. Below, we generalize Theorem 19 in [11] to the multidimensional, radially symmetric situation. Specifically, let $\gamma \in \mathbb{R}$ and $S \in \Sigma_{K}$ be given, and define

$$
\mathbb{I}(u)=\int_{0}^{1} u(r)^{\gamma} \mathrm{D}_{S}[u, r] r^{d-1} \mathrm{~d} r .
$$


Further, define the components of a vector $\bar{\xi} \in \mathbb{R}^{K}$ by

$$
\bar{\xi}_{1}=\sigma, \quad \bar{\xi}_{2}=\sigma(\sigma-1), \ldots, \quad \bar{\xi}_{K}=\sigma(\sigma-1) \cdots(\sigma-K+1),
$$

where $\sigma=(K-d) / \gamma$. By inserting $(\eta, \xi)=(1, \bar{\xi})$ into formula $(2.7)$, one easily verifies that all shift polynomials $T_{k}$ vanish at this particular point. Therefore, the values of any two characteristic symbols $S$ and $S^{\prime}$ coincide at $(1, \bar{\xi})$. Hence, if the given $S$ is negative at $(1, \bar{\xi})$, so is any affine combination $S+c_{1} T_{1}+\cdots+c_{m} T_{m}$. In this case, $\mathbb{I}(u)$ cannot be written as an integral over a pointwise nonnegative expression by the method developed before. This statement can be strengthened as follows.

TheOREm 5.1. Assume that $S(1, \bar{\xi})<0$. Then there exists a family of functions $u_{\varepsilon} \in \mathcal{U}$ with $u_{\varepsilon}(r)=1$ for $r \in[2 / 3,1]$ satisfying $\lim _{\varepsilon \downarrow 0} \mathbb{I}\left(u_{\varepsilon}\right)=-\infty$.

The set $\mathcal{U}$ is defined on page 359 . We remark that, since the functions $u_{\varepsilon}$ are equal to a positive constant for $r>2 / 3$, they satisfy any homogeneous boundary condition that involves derivatives at $r=1$.

The principal idea for our definition of $u_{\varepsilon}$ in (5.1) is borrowed from Laugesen's construction of a "trial function" in one space dimension [17]. Our definition and also the proof of $\mathbb{I}\left(u_{\varepsilon}\right) \rightarrow-\infty$ are more straightforward, since we work under the assumption of strict homogeneity (2.1); the proof in [17] has been designed for a slightly more general situation. The functions $u_{\varepsilon}$ are chosen as suitable $\varepsilon$-regularizations of the radially symmetric power function $\tilde{u}(x)=r^{\sigma}$. A purely formal calculation gives $D_{S}[r, \tilde{u}]=r^{\gamma \sigma-K} S(1, \bar{\xi})$ and, even more formally, $\mathbb{I}(\tilde{u})=S(1, \bar{\xi}) \int_{0}^{1} r^{-1} d r=-\infty$. The rigorous calculations below heavily exploit the marginal singularity of the $r^{-1}$-integral for the estimation of the additional terms that originate from the regularization $\tilde{u} \rightarrow u_{\epsilon}$; the argument would not work for $\sigma \neq(K-d) / \gamma$.

Proof. Let a cut-off function $\phi \in C^{\infty}(\mathbb{R})$ with $0 \leq \phi \leq 1$ be given that satisfies

$$
\phi(r)=1 \quad \text { for } \quad r \leq 1 / 3 \quad \text { and } \quad \phi(r)=0 \text { for } r \geq 2 / 3 .
$$

Choose $\varepsilon \in(0,1 / 2)$ arbitrary and define $u_{\varepsilon}$ by

$$
u_{\varepsilon}(r)=\phi(r / \varepsilon) \varepsilon^{\sigma}+[1-\phi(r / \varepsilon)] \phi(r) r^{\sigma}+1-\phi(r) .
$$

Clearly, $u_{\varepsilon}$ is positive and of class $C^{\infty}$. Moreover, notice that $u_{\varepsilon}(r)=1$ for $2 / 3 \leq r \leq 1$ as stated in the theorem. We need to evaluate the integral

$$
\mathbb{I}\left(u_{\varepsilon}\right)=\int_{0}^{1} u_{\varepsilon}(r)^{\gamma} \mathrm{D}_{P}\left[u_{\varepsilon}, r\right] r^{d-1} \mathrm{~d} r .
$$

This is done by splitting the domain [0,1] into three intervals. To start with, let $r \in$ $[0,2 \varepsilon / 3]$. Then $u_{\varepsilon}(r)=\varepsilon^{\sigma} \psi(r / \varepsilon)$, where $\psi(\rho)=\phi(\rho)+[1-\phi(\rho)] \rho^{\sigma}$, and consequently

$$
\frac{\partial_{r}^{k} u_{\varepsilon}(r)}{u_{\varepsilon}(r)}=\varepsilon^{-k} \frac{\partial_{\rho}^{k} \psi(\rho)}{\psi(\rho)}
$$

with $\rho=r / \varepsilon$. The homogeneity (2.1) of $S \in \Sigma_{K}$ now implies

$$
\mathrm{D}_{S}\left[u_{\varepsilon}, r\right]=\varepsilon^{-K} \mathrm{D}_{S}[\psi, \rho] .
$$

Substitution of $r=\varepsilon \rho$ under the integral leads to

$$
I_{1}:=\int_{0}^{2 \varepsilon / 3} u_{\varepsilon}(r)^{\gamma} \mathrm{D}_{P}\left[u_{\varepsilon}, r\right] r^{d-1} \mathrm{~d} r=\varepsilon^{\sigma \gamma-K+d} \int_{0}^{2 / 3} \psi(\rho)^{\gamma} \mathrm{D}_{P}[\psi, \rho] \rho^{d-1} \mathrm{~d} \rho .
$$


Since $\psi$ is positive and smooth, and all of its derivatives vanish at $\rho=0$, the last integral is well-defined and finite. In fact, the value of $I_{1}$ is independent of $\varepsilon$, since $\sigma \gamma=K-d$ by definition of $\sigma$.

Next, let $r \in[2 \varepsilon / 3,1 / 3]$ and notice that $u_{\varepsilon}(r)=r^{\sigma}$. It follows that

$$
\partial_{r}^{k} u_{\varepsilon}(r)=\sigma(\sigma-1) \cdots(\sigma-k+1) r^{\sigma-k}=r^{-k} \bar{\xi}_{k} u_{\varepsilon}(r)
$$

Using the homogeneity (2.1) once again, we obtain $\mathrm{D}_{S}\left[u_{\varepsilon}, r\right]=r^{-K} S\left(1, \bar{\xi}_{1}, \ldots, \bar{\xi}_{K}\right)$, and thus

$$
\begin{aligned}
I_{2}: & =\int_{2 \varepsilon / 3}^{1 / 3} u_{\varepsilon}(r)^{\gamma} \mathrm{D}_{S}\left[u_{\varepsilon}, r\right] r^{d-1} \mathrm{~d} r \\
& =S\left(1, \bar{\xi}_{1}, \ldots, \bar{\xi}_{K}\right) \int_{2 \varepsilon / 3}^{1 / 3} r^{\gamma \sigma+d-K} \frac{\mathrm{d} r}{r} \\
& =S\left(1, \bar{\xi}_{1}, \ldots, \bar{\xi}_{K}\right) \ln [1 /(2 \varepsilon)] .
\end{aligned}
$$

Finally, for $r \in[1 / 3,1]$, the function $u_{\varepsilon}(r)$ is smooth and positive, and does not depend on $\varepsilon>0$. In other words,

$$
I_{3}:=\int_{1 / 3}^{1} u_{\varepsilon}(r)^{\gamma} \mathrm{D}_{S}\left[u_{\varepsilon}, r\right] r^{d-1} \mathrm{~d} r
$$

is a finite, $\varepsilon$-independent value. In summary, there is some constant $C>0$ for which

$$
\mathbb{I}\left(u_{\varepsilon}\right)=I_{1}+I_{2}+I_{3}=C+S\left(1, \bar{\xi}_{1}, \ldots, \bar{\xi}_{K}\right) \ln [1 /(2 \varepsilon)] .
$$

This sum converges to $-\infty$ as $\varepsilon \downarrow 0$ since $S\left(1, \bar{\xi}_{1}, \ldots, \bar{\xi}_{K}\right)<0$ by assumption.

As a corollary, we obtain that $E_{\alpha}$ cannot be an entropy for the evolution Equation (2.2) if the associated canonical symbol $S_{0}$ has the property that

$$
S_{0}(1, \sigma, \sigma(\sigma-1), \ldots, \sigma(\sigma-1) \cdots(\sigma-K+1))<0
$$

for $\sigma=(K-d) /(\alpha+\beta)$. Indeed, we may use the corresponding function $u_{\varepsilon}$ constructed in the proof of Theorem 5.1 above as an initial condition $u_{0}$ in (2.3). The functions $u_{\varepsilon}$ are positive and smooth, and they satisfy the boundary conditions since $u_{\varepsilon}$ is constant close to the boundary. By classical parabolic theory, there exists a corresponding solution $u_{\varepsilon}(t)$, at least locally in time, i.e. for $t \in[0, \tau]$, and this solution and its spatial derivatives depend continuously on $t \in[0, \tau]$. Hence,

$$
E_{\alpha}\left[u_{\varepsilon}(\tau)\right]-E_{\alpha}\left[u_{\varepsilon}\right]=-\omega_{d} \int_{0}^{\tau} \int_{0}^{1} u_{\varepsilon}(t ; r)^{\gamma} \mathrm{D}_{S_{0}}\left[u_{\varepsilon}(t), r\right] r^{d-1} \mathrm{~d} r \mathrm{~d} t .
$$

Choosing $\varepsilon$ and $\tau$ sufficiently small, the double integral on the right-hand side is negative, and one concludes that $E_{\alpha}\left[u_{\varepsilon}(\tau)\right]>E_{\alpha}\left[u_{\varepsilon}\right]$.

We apply this result to the fourth- and sixth-order equations introduced in the introduction. It turns out that for the thin-film Equation (1.1), we have $S_{0}(1, \bar{\xi})<0$ if and only if $\alpha+\beta \notin[3 / 2,3]$ for $d=1, \alpha+\beta \in(-\infty, 1)$ for $d=2, \alpha+\beta \in(-1,1 / 2)$ for $d=3$, and $\alpha+\beta \in(-(d-4) / 2,(d-4 /(d+2))$ for $d>4$ (our method does not give any statement for $d=4)$. In one space dimension, we achieve the optimal bounds for $\alpha+\beta$, being in the interval $[3 / 2,3]$ (as in $[17,11]$ ). However, we obtain much less information for $d>1$. 
For the DLSS Equation (1.2), $S_{0}(1, \bar{\xi})<0$ holds if and only if $\alpha \notin[0,3 / 2]$ for $d=1$, $\alpha \in(-\infty, 0)$ for $d=2, \alpha \in(-1 / 2,0)$ for $d=3$, and $\alpha \in(0,(d-4) /(2 d-4))$ for $d \geq 4$. We recover the optimal range in the one-dimensional case. Moreover, we see that the lower bound for $d \geq 8$ is optimal, at least for nonnegative values for $\alpha$.

Finally, for the sixth-order Equation $(1.3)$, we have $S_{0}(1, \bar{\xi})<0$ if and only if $\alpha \in(5 / 4,10 / 3)$ for $d=1, \alpha \in(4 / 3, \infty)$ for $d=2, \alpha \notin[-3(1-\sqrt{33}) / 8,-3(1+\sqrt{33}) / 8]$ for $d=3$, and $\alpha \in(-\infty,-1)$ for $d=4$. For higher space dimensions, $S_{0}(1, \bar{\xi}) \geq 0$ holds for all $\alpha \in \mathbb{R}$ and we do not obtain any information. In the two-dimensional case, there are no entropies for $\alpha>4 / 3$, which is not far from the upper bound $\alpha=1.0982 \ldots$ obtained in Theorem 1.3.

Appendix A. In this appendix we give a sketch of the derivation of the sixthorder Equation (1.3). This equation is formally derived from an $O\left(\varepsilon^{6}\right)$ approximation of the generalized quantum drift-diffusion model of Degond et al. [8], where $\varepsilon$ is the scaled Planck constant. Without electric field, this model is given by

$$
\partial_{t} U=\operatorname{div}(U \nabla A),
$$

where the particle density $U(t ; x)$ and the function $A(t ; x)$ are related through the integral

$$
U(t ; x)=\frac{1}{(2 \pi \varepsilon)^{d}} \int_{\mathbb{R}^{d}} \operatorname{Exp}\left(A(t ; x)-\frac{|p|^{2}}{2}\right) \mathrm{d} p, \quad x \in \mathbb{R}^{d}, t>0
$$

Here, the so-called quantum exponential Exp is defined by $\operatorname{Exp}(a)=W\left(\exp \left(W^{-1}(a)\right)\right)$, where $a(t ; x, p)$ is a function in the phase-space, $W$ is the Wigner transform, $W^{-1}$ its inverse and exp is the operator exponential. For precise definitions and the derivation of the quantum drift-diffusion model we refer to [8].

The crucial step in the $O\left(\varepsilon^{6}\right)$ derivation of (A.1) is to determine an $O\left(\varepsilon^{6}\right)$ approximation of $\operatorname{Exp}(a)$ with $a(x, p)=A(t ; x)-|p|^{2} / 2$. To this end, we follow the strategy proposed in [8]. Define $F(z)=\operatorname{Exp}(z a)$ and expand $F(z)$ formally as a series in $\varepsilon$, i.e. $F(z)=\sum_{k=0}^{\infty} \varepsilon^{k} F_{k}(z)$. The functions $F_{k}(z)$ can be computed by pseudo-differential calculus. For odd indices $k$, we have $F_{k}(z)=0$, and for even indices we have to solve the following differential equation:

$$
\frac{d}{d z} F_{k}(z)=a \circ_{0} F_{k}(z)+a \circ_{2} F_{k-2}(z)+\ldots+a \circ_{k} F_{0}(z), \quad z>0,
$$

with the initial condition $F_{k}(0)=\delta_{k 0}$. The multiplication $\circ_{n}$ is defined for any two smooth functions $\omega_{1}$ and $\omega_{2}$ by (see also (5.19) in [8])

$$
\omega_{1} \circ_{n} \omega_{2}=\sum_{|\alpha|+|\beta|=n}\left(\frac{i}{2}\right)^{n} \frac{(-1)^{|\beta|}}{\alpha ! \beta !} \partial_{x}^{\alpha} \partial_{p}^{\beta} \omega_{1} \partial_{x}^{\beta} \partial_{p}^{\alpha} \omega_{2},
$$

where $\alpha, \beta \in \mathbb{N}^{d}$ are multi-indices.

Let $\nabla^{k}$ denote the $k$-tensor of partial derivatives of order $k$, i.e.,

$$
\left(\nabla_{x}^{k} \omega\right)_{i_{1}, i_{2}, \cdots, i_{k}}=\partial_{x}^{\left(i_{1}, i_{2}, \ldots, i_{k}\right)} \omega, \quad\left(\nabla_{p}^{k} \omega\right)_{j_{1}, j_{2}, \cdots, j_{k}}=\partial_{p}^{\left(j_{1}, j_{2}, \ldots, j_{k}\right)} \omega .
$$

Lemma A.1.

$$
\omega_{1} \circ_{n} \omega_{2}=\frac{i^{n}}{2^{n} n !}\left(\sum_{k=0}^{n}(-1)^{k}\left(\begin{array}{l}
n \\
k
\end{array}\right)\left(\nabla_{x}^{n-k} \otimes \nabla_{p}^{k} \omega_{1}\right):\left(\nabla_{p}^{n-k} \otimes \nabla_{x}^{k} \omega_{2}\right)\right),
$$


where " $\otimes$ " denotes the tensor product and ":" the component-wise inner product.

Proof. Let $k=|\beta|=\beta_{1}+\cdots+\beta_{d}$ for $\beta \in \mathbb{N}^{d}$. According to the Schwartz rule, each partial derivative $\partial_{p}^{\beta}$ appears in $\nabla_{p}^{k}$ on exactly $k ! / \beta$ ! positions, where $\beta !=\beta_{1} ! \ldots \beta_{d} !$. Analogusly, for $|\alpha|=n-k$, each $\partial_{x}^{\alpha}$ appears at $\nabla_{x}^{n-k}$ on $(n-k) ! / \alpha$ ! positions. Thus, the expression $\partial_{x}^{\alpha} \partial_{p}^{\beta}$ appears in $\nabla_{x}^{n-k} \otimes \nabla_{p}^{k}$ on $(n-k) ! k ! /(\alpha ! \beta !)$ positions, and the same number of appearances holds for the expression $\partial_{x}^{\alpha} \partial_{p}^{\beta} \partial_{x}^{\beta} \partial_{p}^{\alpha}$ in $\left(\nabla_{x}^{n-k} \otimes \nabla_{p}^{k}\right):\left(\nabla_{p}^{n-k} \otimes \nabla_{x}^{k}\right)$. Using these combinatorial observations, formula (A.3) follows immediately.

The functions $F_{0}(z)$ and $F_{2}(z)$ have already been calculated in [8]:

$$
\begin{aligned}
& F_{0}(z)(x, p)=e^{z a(x, p)}, \\
& F_{2}(z)(x, p)=\frac{1}{8} e^{z a(x, p)}\left(z^{2} \Delta_{x} A+\frac{z^{3}}{3}\left|\nabla_{x} A\right|^{2}-\frac{z^{3}}{3} \nabla_{x}^{2} A: p \otimes p\right) .
\end{aligned}
$$

Thus, it remains to solve

$$
\begin{aligned}
\frac{d}{d z} F_{4}(z)= & a \circ_{0} F_{4}(z)+a \circ_{2} F_{2}(z)+a \circ_{4} F_{0}(z)=a \cdot F_{4}(z) \\
& +\frac{e^{z a}}{192}\left[z^{5}|\nabla A|^{4}+5 z^{4}|\nabla A|^{2} \Delta A-2 z^{5}|\nabla A|^{2}\left(\nabla^{2} A: p \otimes p\right)\right. \\
& -4 z^{4}\left(\nabla^{2} A: \nabla^{2} A p \otimes p\right)+z^{5}\left(\nabla^{2} A: p \otimes p\right)^{2}+2 z^{3}\left\|\nabla^{2} A\right\|^{2} \\
& -5 z^{4} \Delta A\left(\nabla^{2} A: p \otimes p\right)+6 z^{3}(\Delta A)^{2}+3 z^{2} \Delta^{2} A+z^{3} \Delta|\nabla A|^{2} \\
& -z^{3} \Delta\left(\nabla^{2} A: p \otimes p\right)+6 z^{3} \nabla A \cdot \nabla \Delta A+2 z^{4} \nabla A \cdot \nabla|\nabla A|^{2} \\
& \left.-2 z^{4} \nabla A \cdot \nabla\left(\nabla^{2} A: p \otimes p\right)\right]+\frac{\mathbf{e}^{z a}}{384}\left[z^{4}\left(\nabla^{4} A: p \otimes p \otimes p \otimes p\right)\right. \\
& -z^{3}\left(\nabla^{4} A:(p \otimes p \otimes \mathbb{I})\right)-z^{3}\left(\nabla^{4} A: p \otimes \nabla_{p}(p \otimes p)\right) \\
& -z^{3}\left(\nabla^{4} A: \nabla_{p}(p \otimes p \otimes p)\right)+z^{2}\left(\nabla^{4} A: \nabla_{p}(p \otimes \mathbb{I})\right) \\
& \left.+z^{2}\left(\nabla^{4} A: \nabla_{p}^{2}(p \otimes p)\right)\right]
\end{aligned}
$$

with $F_{4}(0)=0$. In the above computations, we have exhaustively used Lemma A.1. By the variation-of-constants formula, we obtain

$$
\begin{aligned}
F_{4}(1)= & \frac{e^{a}}{384}\left[\frac{1}{3}|\nabla A|^{4}+2|\nabla A|^{2} \Delta A-\frac{2}{3}|\nabla A|^{2}\left(\nabla^{2} A: p \otimes p\right)\right. \\
& -\frac{8}{5}\left(\nabla^{2} A: \nabla^{2} A p \otimes p\right)+\frac{1}{3}\left(\nabla^{2} A: p \otimes p\right)^{2}+\left\|\nabla^{2} A\right\|^{2} \\
& -2 \Delta A\left(\nabla^{2} A: p \otimes p\right)+(\Delta A)^{2}+2 \Delta^{2} A+\frac{1}{2} \Delta|\nabla A|^{2} \\
& -\frac{1}{2} \Delta\left(\nabla^{2} A: p \otimes p\right)+3 \nabla A \cdot \nabla \Delta A+\frac{4}{5} \nabla A \cdot \nabla|\nabla A|^{2} \\
& -\frac{4}{5} \nabla A \cdot \nabla\left(\nabla^{2} A: p \otimes p\right)+\frac{1}{5}\left(\nabla^{4} A: p \otimes p \otimes p \otimes p\right) \\
& -\frac{1}{4}\left(\left(\nabla^{4} A:(p \otimes p \otimes \mathbb{I})\right)+\left(\nabla^{4} A: p \otimes \nabla_{p}(p \otimes p)\right)\right. \\
& \left.+\left(\nabla^{4} A: \nabla_{p}(p \otimes p \otimes p)\right)\right)+\frac{1}{3}\left(\left(\nabla^{4} A: \nabla_{p}(p \otimes \mathbb{I})\right)\right. \\
& \left.\left.+\left(\nabla^{4} A: \nabla_{p}^{2}(p \otimes p)\right)\right)\right] .
\end{aligned}
$$


This gives us the $O\left(\varepsilon^{6}\right)$ expansion of the quantum exponential.

It remains to represent the density $u$ as a function of $A$. We integrate $F_{0}, F_{2}$, and $F_{4}$ with respect to $p \in \mathbb{R}^{d}$ and employ the formulas

$$
\begin{aligned}
\frac{1}{(2 \pi \varepsilon)^{d}} \int_{\mathbb{R}^{d}} p_{i} p_{j} e^{A-|p|^{2} / 2} \mathrm{~d} p & =\frac{e^{A}}{(\sqrt{2 \pi} \varepsilon)^{d}} \delta_{i j}, \\
\frac{1}{(2 \pi \varepsilon)^{d}} \int_{\mathbb{R}^{d}} p_{r} p_{s} p_{i} p_{j} e^{A-|p|^{2} / 2} \mathrm{~d} p & =\frac{e^{A}}{(\sqrt{2 \pi} \varepsilon)^{d}}\left(\delta_{r s} \delta_{i j}+\delta_{r i} \delta_{s j}+\delta_{r j} \delta_{s j}\right),
\end{aligned}
$$

where $\delta_{i j}$ denotes the Kronecker symbol. This gives

$$
\begin{aligned}
U= & \frac{1}{(2 \pi \varepsilon)^{d}} \int_{\mathbb{R}^{d}}\left(F_{0}(1)+\varepsilon^{2} F_{2}(1)+\varepsilon^{4} F_{4}(1)\right) \mathrm{d} p+O\left(\varepsilon^{6}\right) \\
= & \frac{e^{A}}{(\sqrt{2 \pi} \varepsilon)^{d}}\left(1+\frac{\varepsilon^{2}}{24}\left(2 \Delta A+|\nabla A|^{2}\right)+\frac{\varepsilon^{4}}{5760}\left(5|\nabla A|^{4}+20|\nabla A|^{2} \Delta A\right.\right. \\
& +\left\|\nabla^{2} A\right\|^{2}+20(\Delta A)^{2}+24 \Delta^{2} A+\frac{15}{2} \Delta|\nabla A|^{2}+33 \nabla A \cdot \nabla \Delta A \\
& \left.\left.+12 \nabla A \cdot \nabla|\nabla A|^{2}\right)\right)+O\left(\varepsilon^{6}\right) .
\end{aligned}
$$

To obtain an $\varepsilon$-expansion of $A$ in terms of $U$, we insert the ansatz $A=A_{0}+\varepsilon^{2} A_{2}+$ $\varepsilon^{4} A_{4}+O\left(\varepsilon^{6}\right)$ in the above expression for $u$. Equating equal powers of $\varepsilon$ yields the system

$$
\begin{aligned}
U= & \frac{e^{A_{0}}}{(\sqrt{2 \pi})^{d}}, \quad 0=A_{2}+\frac{1}{24}\left(2 \Delta A_{0}+\left|\nabla A_{0}\right|^{2}\right), \\
0= & A_{4}+\frac{1}{2} A_{2}^{2}+\frac{1}{24} A_{2}\left(2 \Delta A_{0}+\left|\nabla A_{0}\right|^{2}\right)+\frac{1}{12}\left(\Delta A_{2}+\nabla A_{0} \cdot \nabla A_{2}\right) \\
& +\frac{1}{5760}\left(5\left|\nabla A_{0}\right|^{4}+20\left|\nabla A_{0}\right|^{2} \Delta A_{0}+\left\|\nabla^{2} A_{0}\right\|^{2}+20\left(\Delta A_{0}\right)^{2}\right. \\
& \left.+24 \Delta^{2} A_{0}+\frac{15}{2} \Delta\left|\nabla A_{0}\right|^{2}+33 \nabla A_{0} \cdot \nabla \Delta A_{0}+12 \nabla A_{0} \cdot \nabla\left|\nabla A_{0}\right|^{2}\right) .
\end{aligned}
$$

Therefore,

$$
\begin{aligned}
& A_{0}= \log U+d \log (\sqrt{2 \pi}), \quad A_{2}=-\frac{1}{6} \frac{\Delta \sqrt{U}}{\sqrt{U}}, \\
& A_{4}=\frac{1}{720}\left(2 \frac{\Delta^{2} U}{U}-3 \frac{|\nabla U|^{4}}{u^{4}}+4 \nabla^{2} U \nabla U \cdot \nabla U+4 \frac{\Delta U}{U} \frac{|\nabla U|^{2}}{U^{2}}-4 \frac{\nabla \Delta U}{U} \cdot \frac{\nabla U}{U}\right. \\
&\left.\quad-2\left(\frac{\Delta U}{U}\right)^{2}-\frac{\left\|\nabla^{2} U\right\|^{2}}{U^{2}}\right) \\
&=\frac{1}{360}\left(\frac{1}{2}\left\|\nabla^{2} \log U\right\|^{2}+\frac{1}{U} \nabla^{2}:\left(U \nabla^{2} \log U\right)\right) .
\end{aligned}
$$

Finally, up to terms of order $O\left(\varepsilon^{6}\right)$, (A.1) becomes

$$
\begin{aligned}
\partial_{t} U= & \Delta U-\frac{\varepsilon^{2}}{6} \operatorname{div}\left(U \nabla\left(\frac{\Delta \sqrt{U}}{\sqrt{U}}\right)\right) \\
& +\frac{\varepsilon^{4}}{360} \operatorname{div}\left(U \nabla\left(\frac{1}{2}\left\|\nabla^{2} \log U\right\|^{2}+\frac{1}{U} \nabla^{2}:\left(U \nabla^{2} \log U\right)\right)\right) .
\end{aligned}
$$


coefficients like in Lemma 3.1

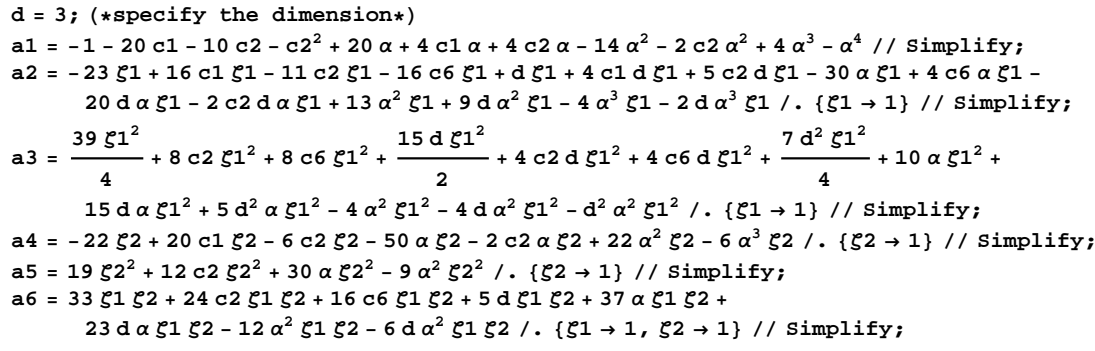

eliminate existence quantifiers

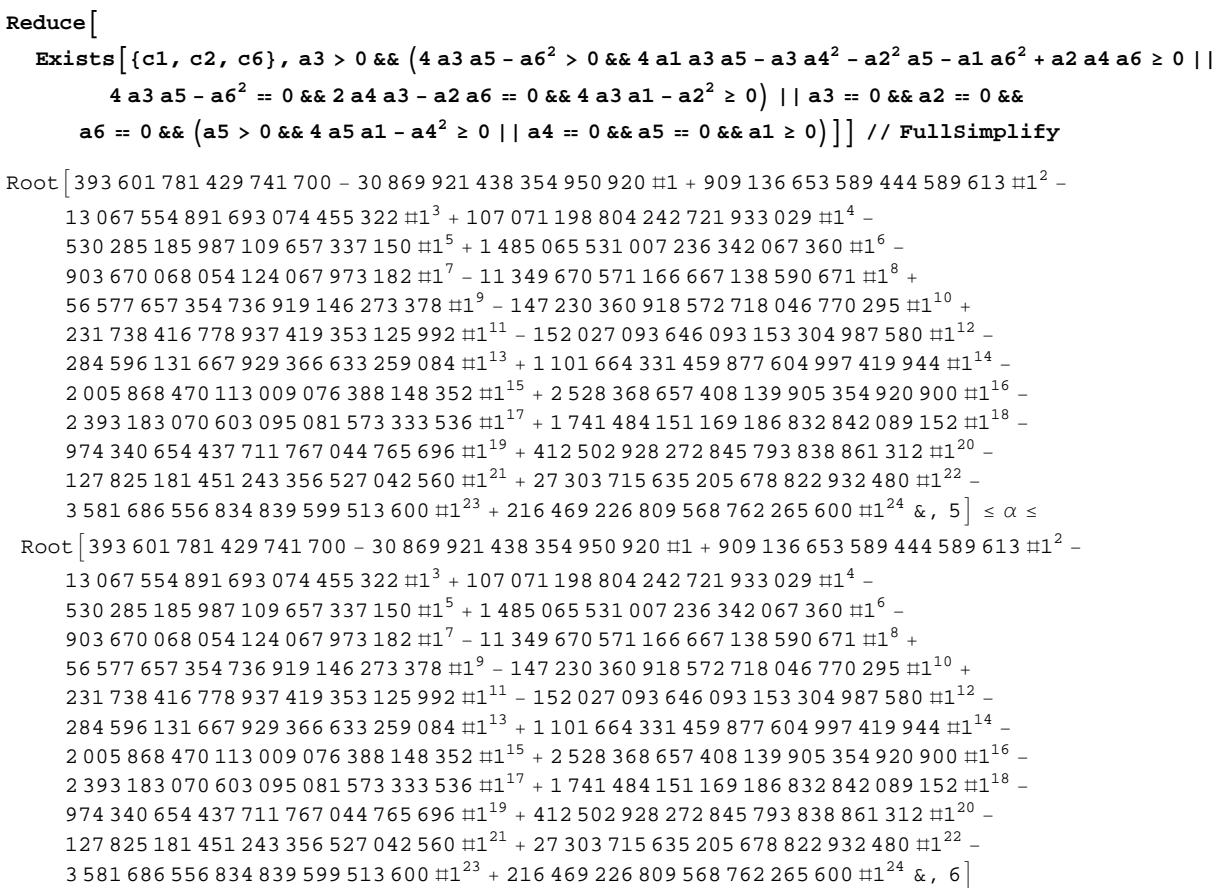

evaluate these roots numerically

$\% / / \mathbf{N}$

$0.347013 \leq \alpha \leq 1.05174$

\section{REFERENCES}

[1] F. Bernis and A. Friedman, Higher order nonlinear degenerate parabolic equations, J. Diff. Eqs., 83, 179-206, 1990.

[2] A. Bertozzi, The mathematics of moving contact lines in thin liquid films, Notices Amer. Math. Soc., 45, 689-697, 1998.

[3] M. Bertsch, R. Dal Passo, H. Garcke and G. Grün, The thin viscous flow equation in higher space dimensions, Adv. Diff. Eqs., 3, 417-440, 1998.

[4] P. Bleher, J. Lebowitz and E. Speer, Existence and positivity of solutions of a fourth-order nonlinear PDE describing interface fluctuations, Commun. Pure Appl. Math., 47, 923942, 1994

[5] S. Basu, R. Pollack and M.F. Roy, Algorithms in Real Algebraic Geometry, Second edition, Algorithms and Computation in Mathematics, Springer, Berlin, 10, 2006.

[6] G. Collins, Quantifier elimination for real closed fields by cylindrical algebraic decomposition, 
Automata Theory and Formal Languages (Second GI Conf., Kaiserslautern, 1975), Lecture Notes in Comput. Sci., Springer, Berlin, 33, 134-183, 1975.

[7] R. Dal Passo, H. Garcke and G. Grün, On a fourth order degenerate parabolic equation: global entropy estimates and qualitative behaviour of solutions, SIAM J. Math. Anal., 29, 321342, 1998.

[8] P. Degond, F. Méhats and C. Ringhofer, Quantum energy-transport and drift-diffusion models, J. Stat. Phys., 118, 625-665, 2005.

[9] B. Derrida, J. Lebowitz, E. Speer and H. Spohn, Fluctuations of a stationary nonequilibrium interface, Phys. Rev. Lett., 67, 165-168, 1991.

[10] U. Gianazza, G. Savaré and G. Toscani, The Wasserstein gradient flow of the Fisher information and the quantum drift-diffusion equation, Arch. Ration. Mech. Anal., 194, 133-220, 2009.

[11] A. Jüngel and D. Matthes, An algorithmic construction of entropies in higher-order nonlinear PDEs, Nonlinearity, 19, 633-659, 2006.

[12] A. Jüngel and D. Matthes, The Derrida-Lebowitz-Speer-Spohn equation: existence, non-uniqueness, and decay rates of the solutions, SIAM J. Math. Anal., 39, 1996-2015, 2008.

[13] A. Jüngel and J.P. Milišić, A sixth-order nonlinear parabolic equation for quantum systems, SIAM J. Math. Anal., 41, 1472-1490, 2009.

[14] A. Jüngel and R. Pinnau, Global non-negative solutions of a nonlinear fourth-order parabolic equation for quantum systems, SIAM J. Math. Anal., 32, 760-777, 2000.

[15] A. Jüngel and I. Violet, Mixed entropy estimates for the porous-medium equation with convection, Discrete Contin. Dynam. Sys. B, 12, 783-796, 2009.

[16] J. King, The isolation oxidation of silicon: the reaction-controlled case, SIAM J. Appl. Math., 49, 1064-1080, 1989.

[17] R. Laugesen, New dissipated energies for the thin film equation, Commun. Pure Appl. Anal., 4, 613-634, 2005.

[18] J. Löfberg, YALMIP: A toolbox for modeling and optimization in MATLAB, Proceedings of the CACSD Conference, Taipei, Taiwan, IEEE, 284-289, 2004.

[19] D. Matthes, A. Jüngel and G. Toscani, Convex Sobolev inequalities derived from entropy dissipation, Arch. Ration. Mech. Anal., 2010.

[20] D. Matthes, R. McCann and G. Savaré, A family of nonlinear fourth order equations of gradient flow type, Commun. Part. Diff. Eqs., 34(10-12), 1352-1397, 2009.

[21] T. Myers, Thin films with high surface tension, SIAM Rev., 40, 441-462, 1998.

[22] A. Tarski, A Decision Method for Elementary Algebra and Geometry, University of California Press, Berkeley, CA, 1951. 